\title{
I. Grundfragen kollektiven Handelns
}

Bereits früh vertrat Tilly (1977) die These einer tiefgreifenden Prägung von Grundfragen kollektiven Handelns durch die Klassiker der Sozialwissenschaften. Dies ist nicht verwunderlich, stellt doch, bei aller gebotenen Vorsicht in der Verengung des klassischen Kanons, die Trias aus Weber, Durkheim und Marx Sozialwissenschaftlern bis heute Narrative spezifischen soziologischen Denkens zur Verfügung (vgl. Baehr 2002), die in sozialwissenschaftlichen Debatten als Allegorien verwendet werden (Kemple 2014: 4ff.). Ihre grundlegenden Schriften liefern bis heute spezifische Komplexe aufeinander bezogener Grundbegriffe, damit verbundene, umfassende Deutungsangebote und nicht zuletzt klassische Formen der Performanz soziologischer Analyse (ebd.).

Gewiss sind die dominanten Konzepte kollektiven Handelns mittlerweile durchaus andere als diejenigen, die Tilly vor Augen hatte. In heute dominanten sozialtheoretischen Konzepten lassen sich dennoch spezifisch zugespitzte Antworten auf Grundfragen wiederfinden, die bereits bei den Klassikern aufgeworfen wurden. Sie bilden daher den ersten Teil der Ausführungen. Die Problemlagen der heute prominenten Heuristiken von James S. Coleman, des WorldPolity-Ansatzes und der ANT spezifiziere ich im Folgenden. Abschließend erfolgt eine knappe Darlegung zentraler Anforderungen und Bezugspunkte für eine sozialtheoretische Heuristik, die sich aus der kritischen Diskussion der drei Traditionen und aktueller Konzepte ergeben.

\section{Grundfragen kollektiven Handelns bei den Klassikern}

Das Befassen mit den Klassikern moderner Sozialwissenschaft ist nicht als eine „bloße Rückschau in die Geschichte der Soziologie, sondern als eine Vergewisserung über Grundprobleme [...] und über Möglichkeiten ihrer Lösung, die uns klarer in die Zukunft [...] sehen läßt“" (Münch 1982: 623), zu verstehen. Dies wird häufig mit Bezug auf die spezifische Persistenz im Funktionieren des Sozialen, aber auch mit der herausragenden praktischen Wirksamkeit von Klassikern 
wie etwa Marx in der gesellschaftlichen Praxis selbst begründet. ${ }^{33}$ Insbesondere können verschiedene Pfade in der Sozialtheorie auf die Rezeption der Klassiker zurückgeführt werden. Einige Grundfragen sind hier außerdem schärfer formuliert worden als in der Nachfolge.

\title{
1.1 Kollektivhandeln, Kollektivgebilde und individuelles Handeln
}

Max Weber fokussiert ein klassisches Thema im Diskurs um das Kollektivhandeln: das Verhältnis zwischen beobachtbaren, handelnden Kollektivgebilden und der Quellen ihrer kollektiven Handlungsfähigkeit im individuellen Handeln. Ganz allgemein geht es ihm um eine prozesshafte Vermittlung von subjektivem Sinn und geteilter Ordnung, die ein kausales Zusammenhandeln ermöglicht. Weber (1972) startet seine Konzipierung des Kollektivhandelns mit Vorstellungen über die Existenz eines Kollektivs, die in einer bestimmten Menschengruppe vorherrschen:

\begin{abstract}
„Die Deutung des Handelns muß von der grundlegend wichtigen Tatsache Notiz nehmen: daß jene dem Alltagsdenken oder dem juristischen (oder anderem Fach-) Denken angehörigen Kollektivgebilde Vorstellungen von etwas teils Seiendem, teils Geltensollendem in den Köpfen realer Menschen (der Richter und Beamten nicht nur, sondern auch des »Publikums «) sind, an denen sich deren Handeln orientiert und daß sie als solche eine ganz gewaltige, oft geradezu beherrschende, kausale Bedeutung für die Art des Ablaufs des Handelns der realen Menschen haben" (ebd.: 7; Herv. RJ).
\end{abstract}

Kollektivgebilde bestehen für Weber also im Kern aus Vorstellungen über für handlungsfähig gehaltene Ordnungen, die dann wiederum ein tatsächliches, kausal wirksames Zusammenhandeln zur Folge haben. Die Grundfrage von Kollektivität stellt sich für Weber wie folgt: Wie verbinden sich die sinnhaften Handlungsorientierung von Subjekten an Kollektivvorstellungen mit dem beobachtbaren, kausalen Wirken von Kollektiven? Es reicht nicht aus, kausal wirksame Gebilde in ihren Wirkungen bloß zu konstatieren. Diese Wirkungen sind ganz im Sinne seiner Vorstellungen moderner Sozialwissenschaften an einen spezifischen subjektiven Sinn gebunden und erst hieraus zu erklären. Genau diese Verknüpfung von einem ähnlich orientierten subjektiv gemeinten Sinn, dem Ausrichten

33 Siehe hierfür etwa Giddens (1984: 348ff.) Argument am Beispiel von Marx und Machiavelli. 
an einer als handlungsfähig angenommenen Kollektivität, begründet auch seine tatsächliche kausale Wirksamkeit.

Man kann hierbei Webers Ausführung weiter in Richtung einer Geteiltheit dieser Handlungsorientierungen unter den am Gebilde Beteiligten deuten:

„Ein moderner ,Staat' besteht zum nicht unerheblichen Teil deshalb in dieser Art: - als Komplex eines spezifischen Zusammenhandelns von Menschen, - weil bestimmte Menschen ihr Handeln an der Vorstellung orientieren, daß er bestehe oder so bestehen solle: daß also Ordnungen von jener juristisch-orientierten Art gelten.“ (ebd.: 7, Herv. RJ)

Kollektives Handeln kann so als Resultat hochgradig ähnlicher und ähnlich aufgenommener Vorstellungen mehrerer Individuen über die Existenz eines Kollektivs angesehen werden. Diese bringen durch ihren Glauben an das Bestehen oder Bestehen-Sollen eines zumindest hochgradig ähnlich durch die Subjekte aufgenommenen Kollektivs auch kollektives Handeln als spezifisches, kausal wirksames Zusammenhandeln erst zustande. Bemerkenswert ist aber, dass die Individuen, indem sie einen hochgradig ähnlichen, subjektiven Sinn verfolgen und die Handlungsfähigkeit eines Gebildes unterstellen, diese auch kausal hervorbringen. Somit verknüpft Weber Kollektivgebilde und tatsächliches Zusammenhandeln über einen ähnlichen, subjektiv gemeinten Sinn der Beteiligten miteinander.

In dem vorgestellten Zitat bezieht Weber Kollektivität weiterhin auf das Gelten einer bestimmten Art oder Qualität von Ordnungen. Was diese Art ausmacht, wird bei Weber jedoch nicht dezidiert geklärt. Im Kern geht es lediglich darum, dass die Ordnungen ein Zusammenhandeln von Menschen zur Folge haben. Diese spärlichen Ausführungen zum Kollektivhandeln wurden in der Weberinterpretation mitunter mit Webers Vorstellungen zum Verband zusammengeführt (Vanberg 1982). ${ }^{34}$ Mitunter wird dabei die angesprochene Spezifik der Ordnung in der Weberinterpretation darin gesehen, dass die Ordnung mit einer zentralen Leitungsinstanz ausgestattet ist. ${ }^{35}$ Dies impliziert auch eine Ein-

34 Auch auf der Andeutung einer juristisch-formalisierten Ordnung beruht der heute prominente Fokus auf stabilen Korporationen als rechtsfähigen Einheiten ganz wesentlich. Eine wesentlich breitere Weberdeutung ist hier jedoch durchaus möglich, wenn nicht gar angebracht.

35 In Webers (1972: 26) Sinne ist ein Verband eine „,nach außen regulierend beschränkte oder geschlossene soziale Beziehung [...], wenn die Innehaltung ihrer Ordnung garantiert wird durch das eigens auf deren Durchführung eingestellte Verhalten bestimmter Menschen: eines Leiters und, eventuell, eines Verwaltungsstabes, der gegebenenfalls normalerweise zugleich Vertretungsgewalt hat". 
grenzung der für handlungsfähig gehaltenen Kollektive, die typisch für eine rationalistische Lesart in der Weberinterpretation ist (siehe Teil I.2.1.).

Hiermit sind bereits bei Weber zentrale Richtungen der Debatte angestoßen: die Abgrenzung zu kollektivem Verhalten über einen ähnlichen subjektiv gemeinten Sinn sowie über eine Ordnungsorientierung, die in der Geltungsannahme jenen ähnlichen subjektiv gemeinten Sinn unter den Beteiligten erst ermöglicht und die Vorstellung eines tatsächlichen, kausalen Zusammenhandelns, das hieraus entsteht und ebenso konstitutiv für kollektives Handeln ist. Kollektivgebilde sind also durchaus im Rahmen der weberschen, sinnorientierten Soziologie zu analysieren. Webers Kritiken an Kollektivbegriffen beziehen sich auf eine spezifische Art und Weise, diese zu thematisieren. Im Rahmen einer handlungstheoretisch orientierten Soziologie ,gibt es [...] keine ,handelnde“ Kollektivpersönlichkeit" (Weber 1972: 6). Seine Skepsis betrifft dabei keineswegs die Vorstellung von Kollektiven generell, sondern vielmehr eine spezifische Fähigkeiten und Quellen des Handelns, die in der Persönlichkeit fußen. ${ }^{36}$ Diese finden sich im Kollektiven nicht. Weber folgend ist also die Frage, wie die Handlungsfähigkeit von Kollektiven verstanden und erklärt werden kann, wenn die Person oder die Persönlichkeit als Faktor ausfällt.

Dieses handlungs- und sinnbezogene Konzept kollektiven Handelns ist weiterhin mit einer spezifischen Vorstellung über die Entstehung, Aufrechterhaltung und Veränderung kollektiven Handelns verknüpft. Weber stellt sich die Konstitutionsfrage vor dem Hintergrund einer Soziologie als Wissenschaft des deutenden Verstehens und damit ursächlichen Erklärens sozialer Phänomene. Kollektive müssen damit notwendigerweise „mikrofundiert“ werden. Auch für die Erklärung kollektiven Handelns gilt also die notwendige Verschränkung von Sinnund Kausaladäquanz, wonach „eine richtige kausale Deutung eines konkreten Handelns bedeutet: daß der äußere Ablauf und das Motiv zutreffend und zugleich in ihrem Zusammenhang sinnhaft verständlich erkannt sind“ (ebd.: 5 ). ${ }^{37}$

36 Webers Kritik richtete sich hier vor allem gegen die damals hoch populäre Massenpsychologie Le Bons' (ebd.: 11). Auch diese Warnungen können als ein klassisches Thema der Debatte um Konzepte kollektiven Handelns gelten. Sie begründen auch schon bei Weber die eingangs beschriebene Forderung nach einer Fundierung des Kollektivhandelns in einem Handlungskonzept. Mit dieser Zurückweisung des Denkens von Le Bon befasst sich die Debatte um kollektives Handeln bis heute (vgl. klassisch für den Bewegungsdiskurs Currie/Skolnick 1970).

37 Der Bezugspunkt eines subjektiv gemeinten Sinns führte Weber dazu, Kollektivitäten stets mit großer Vorsicht zu begegnen und rhetorisch häufig in den Gegenstandsbereich anderer Diszip- 
Wichtig ist es für ihn also, dass auch Kollektive aus ihrer Konstitution im Handeln der Individuen verstanden und in ihrem kausalen Zusammenwirken erklärt werden müssen. ${ }^{38}$

Webers Ansatz zur Erklärung der Entstehung, Aufrechterhaltung und Veränderung kollektiven Handelns geht, wie bereits eingangs beschrieben, von einem subjekt- und sinnbezogenen, zugleich aber prozessual mit der kausalen Produktion von Welt in der Handlungssituation vermittelten Standpunkt aus. Wichtig ist dennoch, zu betonen, dass hier der individuelle Sinnbezug auf eine für handlungsfähig gehaltene Ordnung unabdingbar ist. Erst wenn es einen ähnlichen Bezugspunkt für das kausale Zusammenwirken einer Gruppe gibt, handelt es sich um ein Kollektivhandeln. Die Abgrenzung zu einem Kollektivverhalten wird bei Weber also zentraler Bezugspunkt für die Konzipierung.

linen zu verschieben. Wie Kemple (2014: 226f.) anmerkt, schreibt Weber eine die Sozialwissenschaftler disziplinierende Entgegensetzung in seine Grundlegung der Soziologie ein. Die Soziologie als empirischer Wissenschaft vom subjektiv gemeinten Sinn wird scharf von normativen Gesellschaftswissenschaften abgegrenzt. Letztere befassen sich mit richtigem, sinnvollem, effizientem oder gutem Handeln, in Hinblick auf verschiedene Folgen. „Where specialists in the ,dogmatic sciences of action' may aspire to valid and objective truths or even prescriptive formulas, those in the ,empirical sciences of action' aim to determine ,adequacy' at the level of meaning and ,certainty' with respect to hypothesized motives and probable causes" (ebd.: 227). Mit dieser Trennung versuchte er, die Soziologie gegen die Rechtswissenschaft, Nationalökonomie, aber auch Philosophie zu etablieren. Seine Strategie zielte dabei, mangels eines spezifischen Gegenstandsbereichs, auf den methodologischen Zugriff ab. Dies ist in der Tat dem Vorgehen Durkheims in Frankreich recht ähnlich, nur unter Wahl eines gänzlich anderen Ausgangspunktes: einer durch soziale Ordnungen geprägten Vermittlung von Prozess (Kausalädäquanz) und subjektivem Sinn (Sinnadäquanz) in der Konstitution des Sozialen. Die Frage der Adäquanz hat auch Schütz (1972) zum zentralen Problem sozialwissenschaftlichen Erklärens gemacht. Dabei nimmt er (ebd.: 181) den Zusammenhang von Sinn- und Kausaladäquanz durchaus von Weber her auf, transformiert ihn aber in ein Postulat der Einstimmigkeit der Erfahrung (Schütz1974: 330). Unter Adäquanz versteht er somit den Bezug wissenschaftlicher Konzepte auf als wirklich interpretierte Phänomene (Schütz 1972: 21). All diese Fragen spannen bis heute das interpretative Paradigma der Sozialwissenschaften auf, dem sich auch Giddens Version der Praxistheorie verpflichtet sieht.

38 Nichtsdestoweniger lautet seine eindringliche und häufig vergessene Forderung: „Trotzdem kann die Soziologie auch für ihre Zwecke jene kollektiven Gedankengebilde anderer Betrachtungsweisen nicht etwa ignorieren" (ebd.: 6). Kollektivität muss behandelbar sein, auch (und gerade!) wenn es das vordringlichste Ziel der Soziologie ist, diese in ein Einzelhandeln zu dekonstruieren, da nur so ein Verstehen und somit Erklären des Kollektiven für Weber möglich ist. 


\subsection{Kollektivhandeln, Common Sense und soziale Tatsachen}

Kollektives Handeln wird bei Émile Durkheim anders konzipiert, nämlich im Sinne von „durch die Gesellschaft festgesetzten Verhaltensweisen“ (Durkheim 1984: 100). Es zählt neben den kollektiven Glaubensvorstellungen zum Kernbestand seiner Soziologie, den Institutionen. Kollektives Handeln als äußerlich musterhaft erscheinendes, hochgradig ähnliches und gesellschaftlich akzeptiertes Tun lässt sich hier vor allem als ein Befolgen internalisierter Regeln als Verhaltensnormen verstehen, die dem Individuum zumeist unbewusst, von Zeit zu Zeit schmerzlich bewusst, als äußerliche Zwänge gegenüberstehen (ebd.). Dabei sieht Durkheim durchaus deutlich, dass diese Regeln ganz grundsätzlich menschengemacht sind. Fasziniert zeigt er sich aber vor allem von den sozialen Eigendynamiken, die den unausweichlichen Charakter sozialer Regulierungen ausmachen und sie hinter dem Rücken der Akteure zur Geltung bringen (Durkheim 2002: 85).

Den Kern durkheimschen Denkens bildet eine angenommene Totalität aus kollektiven Klassifikationen und Sozialstrukturen, wobei beide bis zur Unkenntlichkeit miteinander verbunden sind. Die Idee einer kollektiv ähnlichen Aufnahme oder Rahmung der Tatsachen in der Welt, die reale Konsequenzen (im Sinne faktischer Sozialstrukturen) mit sich bringt, bildet das Zentrum seines institutionalistischen Zugriffs. ${ }^{39}$ Die Grundidee bildet die Vorstellung, dass kollektives Handeln aus der Totalität der Gesellschaft selbst heraus erklärt werden muss, da die Gesellschaft sich selbst immer wieder als Totalität reproduziert. Dieser Ausgangspunkt geht in Bezug auf Kollektivität eindrücklich aus den anthropologischen Studien zu primitiven Klassifikationssystemen hervor:

„Society was not simply a model which classificatory thought followed; it was its own divisions which served as divisions for the system of classification. The first logical categories were social categories; the first classes of things were classes of men, into which these things were integrated. It was because men were grouped, and thought of themselves in the form of groups, that in their ideas they grouped other things, and in the beginning the two modes of grouping were merged to the point of being indistinct. Moieties were the first genera; clans, the first species. Things were thought to be integral parts of society, and it was their place in society which determined their place in nature“ (Durkheim/Mauss 2009: 49f., Herv. RJ).

39 Hier spitzen spätere Institutionalisten nur noch auf konkrete, angenommene Tatsachen zu, etwa der World-Polity-Ansatz auf die Akteurswerdung und die Konsequenzen der Zuschreibung eines Akteursstatus. 
Diese Bestimmungen deuten an, dass es ein gruppenbezogenes Denken in der Gesellschaft selbst ist, das Gesellschaft als emergentes Gruppenphänomen erst hervorbringt. Das ist zunächst der weberschen Grundfigur nicht ganz unähnlich. Durkheim zieht an diesem Punkt aber gänzlich andere Schlussfolgerungen: Sozialwissenschaftler müssen dem Kollektivismus der Gesellschaft insofern Rechnung tragen, dass sie auch kollektivbezogene Erklärungen für gesellschaftliche Phänomene finden müssen. ${ }^{40}$ Aus einem eigendynamischen, historischen Prozess entsteht über ähnliche Sozialisation der Gesellschaftsmitglieder ein Kollektivbewusstsein, ${ }^{41}$ das aus der Totalität an „beliefs and sentiments common to the average member of a society“ (Durkheim 1989: 38) besteht.

Im analytischen Zugriff auf kollektives Handeln geht es folglich um die Rekonstruktion einer geteilten Weltsicht, einem Common Sense. Dieser macht das Kollektiv als Kollektiv erst handlungsfähig. Kollektivakteure sind bei Durkheim als mächtige, allumfassende Autoritäten (ebd.: 42) gedacht, die zuvorderst durch den Erhalt und die Entstehung normkonformen Handelns entstehen bzw. sich stabilisieren. Geht es um Kollektivakteure, so betritt bei Durkheim also zumeist die gesellschaftliche Totalität selbst die Bühne. Diese Akteure sind bei ihm als Nationalstaaten verstanden (siehe hierzu Smith 2014: 63ff.). Bei Durkheim stützen sich also Gesellschaften als durch geteiltes Empfinden und Glauben konstituierte Makroakteure und gesellschaftliche Handlungsweisen (im Sinne üblicher Konventionen und Gepflogenheiten) wechselseitig. Kollektive Handlungsweisen und für handlungsfähig gehaltene Kollektive werden, sobald sie einander stützen und Stabilität gewinnen, zu sozialen Tatsachen. Beide nehmen dann einen objektiven und natürlichen Charakter an.

40 Erst vor dem Hintergrund dieser erkenntnistheoretischen Schrift lässt sich also das häufig zitierte durkheimsche Diktum der Erklärung des Sozialen aus dem Sozialen heraus verstehen.

41 Durkheim kennt tatsächlich nur eines, das der Gesellschaft selbst, was Kenneth Smith (2014) ausführlich diskutiert und in eine dem durkheimschen Denken naheliegende Trennung zwischen einem „common consciousness“ und mehreren „collective consciousnesses überführt (ebd.: 15ff.). 


\subsection{Kollektivhandeln, Kampf und die Handlungsfähigkeit in Praxis}

Das klassische Thema kollektiven Handelns bei Marx bezieht sich auf eine spezielle Transformationsproblematik, jene der Klasse an sich in die Klasse für sich:

„Die ökonomischen Verhältnisse haben zuerst die Masse der Bevölkerung in Arbeiter verwandelt. Die Herrschaft des Kapitals hat für diese Masse eine gemeinsame Situation, gemeinsame Interessen geschaffen. So ist diese Masse bereits eine Klasse gegenüber dem Kapital, aber noch nicht für sich selbst. In dem Kampf [...] findet sich diese Masse zusammen, konstituiert sie sich als Klasse für sich selbst. Die Interessen, welche sie verteidigt, werden Klasseninteressen“ (Marx 1977: 180f., Herv. RJ).

Diese Ausführungen zeigen, dass es, wie Negt (1980: 3) zugespitzt kommentiert, bei dieser Transformation um gemeinsame Handlungsfähigkeit geht, was auch immer dies bedeutet:

„Die ,Klasse an sich“ bezeichnet die Gleichheit ökonomischer Bedingungen der Existenz von Millionen von Menschen; daß daraus eine Klasse ,für sich', ein handlungsfähiges Kollektiv wird, liegt weitgehend im Bereich des Subjektiven, setzt zusätzliche und verschiedenartige Erfahrung voraus" (ebd.)

Was nun dieses „Subjektive“ oder die „Erfahrungen“ sind, die die Handlungsfähigkeit des Kollektivs erzeugen, wird bei Marx selbst mit dem Begriff des Kampfes lediglich angedeutet. ${ }^{42}$

Der frühe Marx beschreibt den Prozess selbst als zentralen Ort der Entstehung kollektiven Handelns. Er rückt die aktive Produktion kollektiven Handeln in Praxis ins Zentrum der Aufmerksamkeit. Einzelne Akteure können dabei versuchen, sich durchzusetzen, aber sie sind im Handeln und zum Handeln, insbesondere wenn kollektiv agiert werden soll, auf andere angewiesen. Die wechselseitige Abstimmung dieser verschiedenartig interessierten Akteure muss also selbst im Zentrum der Analyse stehen. Bei Marx betreten in der Analyse konkreter geschichtlicher Entwicklungen die verschiedensten Akteure und Gruppen (auch jenseits sozialer Klassen) die Bühne des Welttheaters, um ein konfliktgeladenes Drama aufzuführen (vgl. Kemple 1995). Kollektives Handeln erwächst

42 Dass die marxistische Tradition in ebenjener Überführung in ein gemeinsames Klassenhandeln eine ihrer großen Lehrstellen hat, wurde schon verschiedentlich kommentiert (prominent in der Debatte um kollektives Handeln bspw. von Bader 1991: 11). 
aus den Beziehungen zwischen ihnen. Auch für Marx' Vorstellung kollektiven Handelns gilt seine grundlegende Bestimmung relationaler und praxisbezogener Sozialität in den Grundrissen:

\begin{abstract}
„Betrachten wir die bürgerliche Gesellschaft im Großen und Ganzen, so erscheint immer als letztes Resultat des gesellschaftlichen Produktionsprozesses die Gesellschaft selbst, d.h. der Mensch selbst in seinen gesellschaftlichen Beziehungen. Alles, was feste Form hat, wie Produkt, etc., erscheint nur als Moment, verschwindendes Moment in dieser Bewegung“ (Marx 1953: 600).
\end{abstract}

Auch Kollektives Handeln erwächst also aus den Beziehungen zwischen den Menschen, die sich in der Praxis selbst zeigen und in ihr beständig (re-)produziert werden. ${ }^{43}$

Deutlich wird diese Konstitution kollektiven Handelns im Prozessieren der Praxis in Marx“ (1960) Analyse der französischen Revolution von 1848. Dieser Text vermag es, den für Marx‘ Verständnis kollektiven Handelns zentralen Unterschied zwischen der Klasse an sich und der Klasse für sich zu illustrieren. ${ }^{44}$

43 Dieses prozessuale und relationale Verständnis von Kollektivität wird bei Marx dennoch immer wieder radikal konterkariert, wenn es zur Beschreibung der Gruppen und Individuen kommt. Hier haben wir es häufig mit relativ einfachen und strukturalistischen Vorstellungen über ein einheitliches Kollektivbewusstsein, etwa im Sinne des Klassenbewusstseins, zu tun. In den Erzählungen wird die eingangs beschriebene, zentrale Transformation hin zu einem handlungsfähigen Kollektiv dabei schlicht vorausgesetzt, oder Potentiale zur Handlungsfähigkeit werden durch die Position in der kapitalistischen Sozialstruktur bestimmt.

44 Ein anderer Aspekt, aus dem der Text seine Popularität bezieht, sind die nahezu seherischen Vorwegnahmen von zentralen Konfliktlinien faschistischer Machtergreifung im 20. Jahrhundert. Wie Marcuse (1965: 143) so passend bemerkt: ,[...] All dies ist zwanzigstes Jahrhundert, aber zwanzigstes Jahrhundert in der Perspektive des neunzehnten, dem das Grauen der faschistischen und nachfaschistischen Periode noch fremd ist." In dieser Passage zeigt sich der analytische Marx, der seine eigene Ideologie an der Wirklichkeit scheitern sieht und dennoch kühl zu analysieren vermag, wie es dazu kam, kommen musste. Marcuse formuliert: „gegen den Willen dessen, der es geschrieben hat, wird das Werk zur großen Literatur. Die Sprache wird zum Begriff der Wirklichkeit, der dem Schrecken des Geschehens durch die Ironie standhält"“ (ebd.: 147). Das ist, was den Ton des Textes ausmacht: Marx versucht die surreale Komik dessen, was passiert, zu fassen, dabei bleibt ihm in jedem Moment das Lachen im Halse stecken. Nur so kann er beschreiben, was passiert, wie es passiert, und das in einer Form, die der Tragödie (vgl. Kemple 1995) ebenso nahesteht wie der bitteren Satire. Anders als Engels (1960: 562) in seinem Vorwort zur dritten Auflage bemerkt, ist es keineswegs so, dass das ,große Bewegungsgesetz der Geschichte [...], wonach alle geschichtlichen Kämpfe [...] in der Tat nur der mehr oder weniger deutliche Ausdruck von Kämpfen gesellschaftlicher Klassen sind, und daß die Existenz und damit auch die Kollisionen dieser Klassen wieder bedingt sind durch den Entwicklungsgrad ihrer ökonomischen Lage, durch die Art und Weise ihrer Produktion und ihres dadurch bedingten Austausches [...] wie das Gesetz von der Verwandlung der Energie für die Naturwissenschaft [... Marx den] Schlüssel zum Verständnis der Geschichte der zweiten 
Marx schreibt hier eine Geschichte der beständig wechselnden Beziehungen zwischen Gruppen, die intendiertes oder geplantes Kollektivhandeln permanent unterlaufen und in ihr faktisch realisiertes Gegenteil verkehren. So schreibt er bspw. bitter über die Bourgeoisie und Religionseliten, die die Umstürze zunächst initiieren:

„Und schließlich werden die Hohenpriester der »Religion und Ordnung« selbst mit Fußtritten von ihren Pythiastühlen verjagt [...]. Ordnungsfanatische Bourgeois auf ihren Balkonen werden von besoffenen Soldatenhaufen zusammengeschossen, ihr Familienheiligtum wird entweiht, ihre Häuser werden zum Zeitvertreib bombardiert - im Namen des Eigentums, der Familie, der Religion und der Ordnung“ (Marx 1960: 123).

Die agierenden Interessengruppen fallen den eigens in Gang gesetzten Prozessen selbst zum Opfer, werden von ebenjenem Strom der Praxis gewaltsam fortgerissen, den sie selbst noch eben in Gang setzten. Jenseits einer universellmaterialistischen Geschichtsschreibung rekonstruiert er die konkrete Abfolge rekursiv miteinander verknüpfter Prozesse. Handlungsfähigkeit erwächst hier letztlich aus der Relationierung verschiedener Handelnder im Prozess selbst mit den ihn ermöglichenden und beschränkenden Bedingungen. Gleichzeitig sind die auftretenden Interessengruppen durchaus klassisch an materiellem Wohlstand interessierte, strategische Akteure, die kämpferisch ihre Interessen durchzusetzen suchen.

Auf diesem sowohl prozessualen als auch strategisch-politischen Konstitutionsverständnis aufbauend haben Autoren eines praxisbezogenen Marxismus weiterhin den Zusammenhang von „Erkenntnis und Interesse“ (Habermas 1968) betont und gezeigt, dass auch die Begriffe der Soziologie, an einen konkreten, historischen Entstehungszusammenhang gebunden sind (Habermas 1978: 238). ${ }^{45}$ Aus den marxschen Frühschriften kann man dabei nicht nur folgern, dass die Soziologie durch das Herauslösen einzelner Elemente aus dem konkreten Entstehungszusammenhang nicht nur den Gegenstand, sondern auch die praktischen

französischen Republik“ gab. Vielmehr transzendiert Marx hier beständig den politökonomischen Strukturdeterminismus seiner späteren Schriften, um die Spezifität der historischen Ereignisse fassen und adäquat analysieren zu können.

45 So handelt es sich bspw. bei der Rolle um keine „universalhistorische Kategorie“ (ebd. 239), und Wissenschaft stellt keine Sphäre objektiven Wissens dar, was eine Auflösung der Aufteilung in Subjekt und Objekt bzw. einem herausgehobenen Wahrheitsanspruch der Wissenschaft zur Folge haben müsste. 
Folgen ihres Tuns aus dem Blick (ebd. 240) verliert. Es folgt daraus ferner, dass man jede gesellschaftliche Realität, die mit theoretischen Mitteln zugänglich gemacht wird, als konkrete Praxis verstehen (ebd.: 241) und das praktische Herstellen, das Wie des Orchestrierens und Mobilisierens von Individuen und Gruppen hin zu einer Intervention in Praxis, analytisch fokussieren muss. ${ }^{46}$

\section{Grundprobleme aktueller Theorien kollektiven Handelns}

Die hier eingeführten Grundfragen nach der Verbindlichkeit eines Kollektivgebildes für das individuelle Handeln (Weber), der, auf einer geteilten Weltsicht fußenden, gesellschaftlichen Klassifikation als Kollektivhandeln (Durkheim) und einer wechselseitigen Mobilisierung zur Transformation der Praxis (Marx) wurden in der Folge immer wieder aufgegriffen. In den heute prominenten Heuristiken kollektiven Handelns wurden diese Grundfragen der Klassiker dabei, sei es in Zustimmung oder Ablehnung, durch das vielschichtige Werk von Talcott Parsons gebrochen. In Bezug auf das Kollektivhandeln sind insbesondere seine Ausführungen zur Bindung an „Collectivities“, aber auch sein, in der Einleitung eingeführter, über Einheitlichkeit bestimmter Begriff des Akteurs zentrale Bezugspunkte, ohne die sich die im Folgenden kritisch diskutierten Engführungen der Grundfragen nicht verstehen lassen.

Parsons' Figur der Kollektivität kann hierbei, wie bereits in der Einleitung beschrieben, als eine über Wertstandards vermittelte Form affektiver Bindung an eine kollektive Identität beschrieben werden. Mit der Bindung führt Parsons ein zentrales Thema in den Diskurs um kollektives Handeln ein. Erst über die Bindung an Kollektivitäten ist eigene Identitätsbildung möglich. Er steht dabei auf den Schultern Durkheims:

„Considering that we are talking about the conditions of relatively stable interaction in social systems, it follows from this that the value-standards which define institutionalized roleexpectations assume to a greater or less degree a moral significance. Conformity with them becomes $[. .$.$] to some degree a matter of the fulfillment of obligations which ego carries relative$ to the interests of the larger action system in which he is involved [...]. The sharing of such common value patterns, entailing a sense of responsibility for the fulfillment of obligations, then creates a solidarity among those mutually oriented to the common values. The actors con- 
cerned will be said to constitute, within the area of relevance of these values, a collectivity" (Parsons 1991: 26, Herv. RJ).

Bei Parsons' Kollektiven geht es um moralisch integrierte Handlungskontexte, Kontexte für deren Funktionieren sich die Beteiligten qua Sozialisation verantwortlich fühlen. Diese geteilte Verantwortlichkeit wiederum führt zu wechselseitiger Solidarität zwischen den am Kollektiv Beteiligten, die die Bindung des Individuums an das Kollektiv nochmals verstärkt. Parsons entwickelt damit ein ganz spezifisch enggeführtes Verständnis der Bindung an Kollektivitäten, das sich, wie später noch deutlich wird, von den deutlich allgemeineren Konzepten der Bindung zwischen Aktivitäten bei Luhmann und Giddens, auf die in der hier vertreten Fassung kollektiven Handelns zurückgegriffen wird, unterscheidet.

Die handlungstheoretische Konzeption des Kollektivhandelns als Handeln einer Korporation nimmt zentrale Themen der Akteurshaftigkeit und Bindung an Kollektivitäten aus dem Werke Parsons auf, versucht diese aber aus einer Mikrofundierung heraus zu erklären. Noch grundlegender fällt die Kritik des WorldPolity-Ansatzes aus. Hier wird vor das parsonssche tiefgreifende Commitment mit einer Kollektivität die Frage nach denjenigen gesellschaftlichen Dynamiken gestellt, die zu einer kognitiven Naturalisierung von Identitäten führen, die für kollektiv handlungsfähig gehalten werden. Ähnlich grundlegend fällt die Kritik der ANT aus, die sich zwar mit Durkheim einen anderen symbolischen Reibungspunkt wählt, in der Sache aber auch an Parsons Ähnliches zu kritisieren hat. Hier steht eine radikale Hinwendung zum Prozess und zur Mobilisierung konkreten Zusammenwirkens im Zentrum. Diese Theoriebewegung versucht sich in dieser Hinwendung zudem von stark sozialisations- und wissensbezogenen Argumenten zu lösen.

\subsection{Kollektivhandeln, vertragsbasierte Systeme und der Korporativakteur}

(A) Kollektivhandeln als Handeln korporativer Akteure

Coleman (1990) definiert kollektives Handeln als korporatives Handeln, als Handeln im Interesse eines Korporativakteurs, und grenzt es dezidiert von kol- 
lektivem Verhalten und von Koordinationsphänomenen im Allgemeinen ab. ${ }^{47} \mathrm{Er}$ behandelt Korporativakteure, also Organisationen und Staaten (ebd.: 333), als in modernen Gesellschaften hochgradig bedeutsame Gebilde. ${ }^{48}$ Seine zentrale Forderung lautet, korporative Akteure als zentrale Akteure moderner Gesellschaften in der Handlungstheorie zu verorten und ihnen dieselben Eigenschaften als Akteure zu Handeln zuzuschreiben, wie Individuen:

„[...] a social theory of purposive action must take as its elementary actors both natural persons and corporate bodies, because both have the essential properties of actors: control over resources and events, interests in resources and events, and the capability of taking actions to realize those interests through that control." (ebd.: 542)

Coleman entwickelt also seine Theorie kollektiven Handelns als Handeln einer Korporation aus einer Übertragung von Eigenschaften individueller Akteure. Die drei hier genannten Eigenschaften ist folglich dann von kollektivem Handeln zu sprechen, wenn ein Sozialsystem als Austauschsystem zwischen Akteuren eigenständige Formen der Kontrolle über die Ressourcen und Situationen auszuüben

47 Die Terminologie des korporativen Handelns wird nicht nur mit kollektivem Handeln gleichgesetzt, sondern diesem sogar vorgezogen, um das Gebunden-Sein an individuelle Entscheidungen zu betonen (vgl. Vanberg 1982: 3, siehe auch Brunner/Meckling 1977). Betont wird: Es gibt im Prinzip kein kollektives Entscheiden, nur ein individuelles im Sinne einer umfassenden Körperschaft bzw. Korporation. Hier wird vor allem die schon bei Weber vorhandene Abgrenzung zu kollektivem Verhalten als unorganisiertem oder spontanem Tun genutzt, um kollektives Handeln als organisiertes Handeln zu kennzeichnen (Vanberg 1982: 8). Mitunter wird diese Gleichsetzung in dieser Tradition auch wieder aufgelöst und zwischen kollektivem Handeln im Sinne eines organisierten Handelns, das an individuelle Entscheidungen gekoppelt ist, und korporativem Handeln im Sinne von Kontexten, in denen ,strategische Entscheidungen abgekoppelt von individuellen Präferenzen der Mitglieder" (Dolata/Schrape 2014: 9) sind, unterschieden. Auch hier ist kollektives Handeln aber an stabile Kollektivakteure geknüpft. Zentrale Kritikpunkte meiner Argumentation sind also von dieser weiteren Differenzierung unabhängig.

48 Im Folgenden beziehe ich mich ausschließlich auf die sozialtheoretische Diskussion der Konstitution von Korporationen und lasse die gesellschaftstheoretischen Argumente (ebd.: 531, Coleman 1986b) beiseite, da sie mir im Kern eine normativ-politische Agenda zu verfolgen scheinen: die Kritik am Bedeutungsverlust des Individuums aus einer libertären Weltanschauung heraus. Was damit im Folgenden nur angedeutet wird, aber nicht vergessen werden darf: Coleman denkt die Korporation nicht nur aus seiner inneren Verfasstheit, sondern ebenso aus seinem gesellschaftlichen Konstruiert-Sein, den Zuschreibungen eines Akteursstatus (insbesondere im Recht) heraus. Die Spezifik des handlungstheoretischen Ansatzes entfaltet sich meines Erachtens jedoch auf der Innenseite, insbesondere in der Theorie des korporativen Selbst. Die Außenseite ist zudem sozialtheoretisch unbestimmt und wird mit gesellschaftstheoretischen Klärungen abgefunden. 
in der Lage ist, hierbei eigene Interessen verfolgt und so eigenständig in der Lage ist Handlungsgelegenheiten wahrzunehmen.

Einführend beschreibt er den Paradefall ${ }^{49}$ der Entstehung einer Korporation aus einer ganz elementaren Situation heraus:

„[...] three individuals agree to create a new actor with the right to act for all. I will call this new actor a corporate actor, for it is a corporate entity which holds rights vested in it by its members. [...] The newly created corporate actor can be seen as the superordinate, and the members who have voluntarily given up to the corporate actor certain rights to control or constrain their actions can be seen as the subordinates. This kind of authority system is a special case, however; since the members retain the right to control the actions of the corporate actor (for example, through collective decisions in which each has voting rights), they have created an actor with authority over them but whose actions they jointly have authority over. It is both superordinate over them and subordinate to them" (ebd.: 330, Herv. RJ).

Korporatives Handeln ist durch ein spezifisches, handlungsfähiges Austauschsystem gekennzeichnet. Im korporativen Handeln stimmen die Beteiligten einer bestimmten Satzung, einem Vertrag zu und überantworten der Korporation einen bestimmten Anteil ihrer Ressourcen. Folglich ist korporatives Handeln durch vertraglich regulierte Formen des Ressourcenpooling unter Schaffung eines von individuellen Präferenzen unabhängigen (und doch von individuellem Handeln erzeugten) kollektiven Selbst gekennzeichnet (ebd.: 509f.). Diesem Kollektivselbst werden die Kontrollrechte an den individuellen Ressourcen von den Individuen erst zugesprochen. Folglich können diese Kontrollrechte der Korporation im Prinzip jederzeit wieder entzogen werden, gleichwohl mit ganz verschiedenen Kosten für die Beteiligten. Die Entstehung eines vertraglich regulierten und kontrollierbaren Ressourcenpools zum Verwirklichen kollektiver Interessen wird

49 Es handelt sich um eine sogenannte „,conjoint constitution“ der Korporation, einen Vertrag um das eigene Handeln eines Handlungszusammenhangs zu bündeln und kontrollieren zu können. Dieses Beispiel verdeutlicht die Grundlogik sehr anschaulich. Coleman stellt dieser Form, auf einem Kontinuum, das auch Zwischengrade kennt (ebd.: 327), die sogenannte ,disjoint constitution“ gegenüber. Diese bezeichnet einen Vertrag, der das Handeln an der Aushandlung des Vertrages nicht beteiligter Dritter zu regulieren versucht. Diesen Fall, den Coleman selbst als praktisch bedeutsamer ansieht (ebd.), diskutiert er etwa am Beispiel amerikanischer Schulen (ebd.: 349ff.). Hier schaffen Lehrer, Eltern und Politiker die Verfasstheit eines Handlungszusammenhangs der für Schüler, die selbst nicht an den Aushandlungen beteiligt werden, zu einer hochgradig verbindlichen Handlungsorientierung wird. Die folgenden Ausführungen beziehen sich auf beide Formate. 
also zum zentralen Merkmal um kollektiv handlungsfähige Sozialsystem von anderen Systemen abgrenzen zu können. ${ }^{50}$

Der Vertrag meint hierbei keineswegs nur eine formale Ordnung, sondern vielmehr eine spezifische Verbindung aus formellen und informellen Regelungen (ebd.: 327). Diese Vorstellung geht zurück auf vertragstheoretische Vorstellungen in der politischen Philosophie, etwa bei Locke (ebd.: 55). Die prinzipielle und interessierte Zustimmung der Mitglieder zur Teilnahme geht einher mit einer partikularen Unterordnung individueller Interessen unter die Kollektivinteressen der Korporation. ${ }^{51}$ Korporatives Handeln ist also eines, in dem ein Kollektiv Ressourcen verschiedener Individuen derart bündeln und kontrollieren kann, dass es als einheitlicher Akteur mit eigenen, korporativen Interessen aufzutreten vermag. Kollektives Handeln wird also vor allem über die Möglichkeit zum Handeln nach kollektiven Interessen bestimmt. Hier zeigt sich, dass Coleman kollektives Handeln und Kollektivakteure radikal zusammendenkt. Korporative Akteure sind jene speziellen „Kollektivgebilde, die als Handlungseinheiten angesprochen werden“(Vanberg 1982: 8, Herv. RJ) können.

Die Konstitution kollektiven Handelns über Verträge und interessierte Individuen

Die Spezifik der Entstehung der drei, im Eingangs vorgestellten Zitat eingeführten, Eigenschaften des Handelns von Akteuren wird für korporative Akteure bei Coleman jeweils spezifisch diskutiert. Individuelles und kollektives Handeln sind für ihn zu einem gewissen Grade parallel und zugleich andersartig verfasst:

50 Nicht jedem Sozialsystem wohnt für Coleman diese Qualität inne (siehe die Typisierung von Austauschsystemen in Coleman 1990: 35). Die aus dieser Einwilligung in den Vertrag entstehende und von den Individuen (in gewisser Weise) unabhängige Kontrollierbarkeit der Korporation grenzt es von einem unkontrollierbaren Kollektivverhalten ab (ebd.: 197ff.).

51 „Korporative Akteure entstehen dadurch, dass Akteure Ressourcen in einen Pool einbringen. Über diesen [...] wird dann nicht mehr individuell, sondern im Verbund disponiert. Und aus der gemeinsamen Disposition über die zusammengelegten Ressourcen ergibt sich ein Kooperationsertrag, der dann auf die individuellen Akteure verteilt werden kann" (Preissendörfer 2005: 28, siehe auch Vanberg 1982). Das hat verschiedene Konsequenzen: Zum einen muss sowohl über die Form des Pooling als auch der Form der Kontrolle über den Pool und die Beteiligung der Mitglieder vertraglich Klarheit hergestellt werden. Zum anderen kommt es über diesen Vertrag zu einer Differenz zwischen dem Handeln im Sinne des Kollektivakteurs und dem Handeln im Sinne der eigenen, individuellen Interessen. 
(i) Von zentraler Bedeutung für kollektives Handeln ist die Orientierung an der Verwirklichung des Interesses einer Korporation. Coleman nimmt in der Konzipierung dieses Interesses die webersche Vorsicht vor der Kollektivpersönlichkeit in spezifischer Weise auf. Über einen kollektiv verbindlichen Vertrag kommt es vor allem zu einer Differenz zwischen dem Handeln im Sinne der Interessen eines Kollektivakteurs und einem Handeln das dem Kollektivakteur aus einem Eigeninteresse überantwortet wurde, damit diese Interessen realisiert werden können. Unter Verweis auf die PrinzipalAgenten-Problematik im amerikanischen Recht (ebd.: 146ff.) beschreibt er diese Differenz als im Handeln korporativer Akteure spezifisch ausgestalteter Beziehung zwischen dem Handlungs- und Objektselbst:

\footnotetext{
„In corporate actors this separation of the self corresponds to physically different parties: the principals (members or owners) as the object self, and the agents (officers or employees) as the acting self. [...] This in turn implies that the concept of interest plays two roles in the theory. For the object self, interests indicate the levels of satisfaction contingent on the outcomes of certain events or control of certain resources. For the acting self, interests indicate the relative amounts of resources that will be committed to gaining control over the event; they serve as the driving forces for action" (ebd.: 509).
}

Das Verhältnis von Handlungs- und Objektselbst erfährt im Kollektivhandeln folglich eine Qualität, die folgenreich für eine Konzipierung kollektiver Interessen ist. Man kann die Konstitution von kollektiven Interessen, dem Zitat folgend, als einen Vermittlungsprozess verstehen. Vermittelt wird zwischen den genuinen Interessen der Korporation als Objektselbst, etwa den Gewinnzielen einer Unternehmung, und den an eigenen Interessen orientierten Investitionsentscheidungen derer, die die Verwirklichung dieses Interesses umsetzen, etwa die Mitarbeiter der Unternehmung. Für diesen Vermittlungsprozess sind wiederrum die bereits eingeführten, vertraglichen Regelungen von zentraler Bedeutung, in denen Rechte und Pflichten festgelegt werden.

(ii) Das Interesse an der Kontrolle von etwas, das Andere kontrollieren, schafft allgemein Handlungssysteme (ebd.: 29). Sozialsysteme entstehen im Allgemeinen durch wechselseitige Abhängigkeiten in der Verfolgung von Interessen und vor allem durch Tausch von Kontrollrechten. Im Handeln von Korporationen werden Ressourcen in der Form zusammengelegt, dass sie zur Verfolgung des Korporationsinteresses kontrolliert werden. Dazu müssen zunächst individuelle Kontrollrechte vertraglich reguliert an eine eigenständige Korporation überantwortet werden. Hierbei orientieren sich nut- 
zenmaximierende Individuen an der Ausgestaltung des Vertrages, insbesondere der Verteilung der Erträge, der internen Regulation der Korporation sowie den Verpflichtungen, die sie eingehen (Vanberg 1982). Erst wenn individuelle Akteure unter Rekurs auf die vertraglichen Ordnung die Verfügungsrechte an einem Teil ihres Tuns an die Korporation abtreten und ihr Tun durch die Korporation kontrollierbar wir, haben wir es mit kollektivem Handeln zu tun.

(iii) Korporationen erlangen durch ihre kollektiven Interessen und den kontrollierbaren Ressourcenpool eine steuerbare, eigene Handlungsfähigkeit. Hier fällt die spezifische Zuspitzung von Webers Frage nach der Verbindlichkeit einer für handlungsfähig gehaltenen Ordnung für das individuelle Handeln ins Auge. Sie erfolgt in Richtung einer vertragstheoretisch gedachten Verbindlichkeit einer Ordnung für die Beteiligten sowie der zentralisierten Steuerungsfähigkeit verschiedener Handlungen durch und über ein kollektives Interesse, das stets mit den Interessen der selbst interessiert-nutzenmaximierend Handelnden Beteiligten vermittelt wird.

Die Konstitution dieser drei Aspekte kollektiven Handelns muss meines Erachtens zudem prozessual und unter Rekurs auf die drei colemanschen Transitionen seiner „Badewanne“ konzipiert werden (Coleman 1990: 8ff.). Coleman hat jedoch die Übertragung seiner Prozessfigur kollektiver Phänomene auf korporatives Handeln meines Wissens selbst jedoch nie explizit gemacht. Mit seiner Badewanne nimmt Coleman dabei Webers Grundidee einer Verknüpfung von Sinnund Kausaladäquanz sowie der Mikrofundierung in ganz spezifischer Art und Weise auf. Indem er Sinn und kausales Wirken, die bei Weber noch zwei Seiten einer Medaille waren, prozessual auseinanderzieht und in ein Makro-MikroMikro-Makro-Modell überführt. Coleman, der sich explizit als methodologischer Individualist versteht und auf Annahmen der Nutzenmaximierung aufbaut, will mit diesem Modell das Verhalten von jedweden Sozialsystemen erklären. ${ }^{52}$ Folg-

52 Hier zeigt sich deutlich, dass es vor allem Talcott Parsons ist, auf dessen Schultern er steht. Colemans theoretisches Hauptwerk ist keineswegs zufällig seinem akademischen Lehrer Robert K. Merton gewidmet, über den er viele der parsonsschen Problemstellungen, aber auch Parsons herausragend systematischen Stil aufnahm (vgl. hierzu Lindenberg 2000). Viele der Mehrdeutigkeiten und lesbaren Unsicherheiten im Werke Webers sind in der Rezeption seines Systematisierers Parsons hierbei geschärft, aber ebenso geglättet. Auch die hochgradig konstruktive und kreative Form der Übersetzung des Werkes Webers durch Parsons ging keines- 
lich müssten auch korporative Akteure (als spezifische Sozialsysteme) dieser Logik des Erklärens folgen. Nimmt man die vorab diskutierten Spezifika kollektiven Handeln als Handeln einer Korporation auf, so ergeben sich drei Transitionen in seiner Konstitution.

In der ersten Transition von der Makro- zur Mikroebene nehmen interessierte Akteure ihre Handlungsbedingungen, vor allem von gesellschaftlich gültigen Werten und Normen, unter Rekurs auf ihren individuellen Nutzen auf. Die Überführung kollektiver in individuelle Normen und Werte ist dabei Resultat einer Vermittlung zwischen kulturell-gesellschaftlicher Prägung, kognitiver Rahmung und der subjektiven Einschätzung der individuellen Situation. Soll es zu einem Kollektivhandeln kommen, so muss hierbei das spezifische Interesse und die vertragliche Ordnung sowie der individuelle Ertrag durch die Beteiligung an einer Korporation eine relevante Handlungsorientierung für den Akteur sein. Es muss den Beteiligten möglich sein die Situation vor dem Hintergrund der korporativen Interessen sowie in der Korporation gültiger Rechte und Pflichten aufzunehmen, sowie dies in Verbindung mit dem eigenen Nutzen zu setzen.

In der zweiten Transition überführen die Akteure ihre Interessen in ein Handeln. Es geht im colemanschen Verständnis um die nutzenmaximierende Entscheidung für eine konkrete Handlung vor dem Hintergrund möglicher Alternativen. Damit es zu korporativem Handeln kommt müssen sich also relevante Akteure aktiv zur Teilhabe am korporativen Akteur entscheiden und der Korporation, unter vertraglich fixierten Bedingungen und in Erwartung eines eigenen Ertrages, einen Teil ihrer Ressourcen überantworten. Individuen entscheiden sich anhand einer rational-kalkulierten Nutzenfunktion dazu in ausgewählten Bereichen dafür ihr Tun in den Dienst eines Kollektivinteresses zu stellen. Die vertragliche Ordnung bildet dabei eine zentrale Orientierung, da sie Einsatz und Ertrag der Beteiligung an der Korporation reguliert.

Die dritte Transition bildet eine Aggregation vertraglich regulierter Handlungen zu einem korporativen Output. Vertraglich reguliert und jeweils rational

wegs ohne Bedeutungsverluste ab. Ein besonders eindrückliches Beispiel hierfür ist, wie Baehr (2002) zeigt, das Bild der Moderne als stahlhartes Gehäuse, das bei Parsons ‘ Übersetzung zum „,iron cage“ wird, was unter anderem seine schützende und Prozesse auch ermöglichende Natur komplett außer Acht lässt. Aus einem Prozess, den Weber so durchaus ambivalent auf den Begriff zu bringen suchte, wurde in Parsons ' Worten in der amerikanischen Tradition ein eindeutig für das Individuum problematischer „,iron cage“. Diese Eindeutigkeiten dagegen wurden interessanterweise in der Kritischen Theorie mitunter in den deutschen Diskurs eingebracht. 
am individuellen Nutzen orientiert greifen Handlungen dann ineinander, erzeugt einen bestimmten kausalen Effekt, der vor dem Hintergrund des Kollektivinteresses verstanden wird. Dies heißt dabei nicht, dass derartige Aggregationen nicht zugleich Wirkungen haben können, die diesem Kollektivinteresse zuwider laufen.

Man kann also von einer spezifizierten „Badewanne“ kollektiven Handelns ausgehen und von spezifischen Sozialsystemen, die wechselseitige Abhängigkeit in Richtung eines aggregierten Outputs im Sinne des Kollektivinteresses motivieren und ausgestalten. Kollektives Handeln wird eine Frage des Erzeugens von Verbindlichkeit in der Aufnahme einer Korporationsordnung, einer individuellen Motivation zum Ressourcenpooling sowie der konzertierten Abstimmung individueller Handlungen in Richtung einer tatsächlichen Erfüllung dieser Interessen.

(C) Eine klare und in einem Handlungskonzept fundierte Heuristik des Korporativakteurs

Coleman greift vor allem die von Weber formulierten Grundfragen nach dem Verhältnis einer verbindlichen Sozialordnung und dem individuellen Handeln in spezifischer Art und Weise auf. Anders als Weber bietet Coleman dabei eine klare Definition des Kollektivhandelns als Handeln einer Korporation an, die zudem explizit in einem Handlungskonzept fundiert wird. Überdies liefert das Konzept mit den drei Aspekten des Handelns als Korporation eine Differenzierung verschiedener Bezugspunkten für einen analytischen Zugriff auf kollektives Handeln an. Eine Abgrenzung zu kollektivem Verhalten und koordiniertem Handeln ist klar konturiert, und auch aus diesen Abgrenzungen werden analytische Bezugspunkte gewonnen.

Weiterhin werden die bereits bei Weber angedeuteten Spezifika jener Ordnungen und Sozialsysteme, die kollektiv-handlungsfähig sind, vertragstheoretisch spezifiziert. Hier steht vor allem die formal-hierarchisch integrierte Organisation Pate, die Weber idealtypisch in seinem Bürokratiemodell diskutiert hat. ${ }^{53}$ Zudem findet sich gar eine (hier bislang nicht explizit diskutierte) gesellschafts-

53 Vanberg (1982) hat, auf Coleman aufbauend, darüber hinaus noch eine Art genossenschaftlichdezentrale Leitung diskutiert. 
theoretische Makrofundierung, die die herausragende Position von organisationalen Korporativakteuren aufnimmt (Coleman 1986b) und diese in Beziehung zur internen Mikrofundierung setzt (Coleman 1990: 325ff.). Insgesamt wird also vor allem Organisationen (und auch Nationalstaaten) als den herausragend bedeutsamen Aktionszentren moderner Gesellschaften eine handlungstheoretische Fundierung gegeben.

(D) Theoretische Herausforderungen des rationalistischen Zugriffs

Colemans Verknüpfung von Kollektivhandeln und Korporation bzw. Kollektivakteur ist problematisch. Sicher können auch mit Coleman die in der Einleitung skizzierten, temporären und fluiden Formen des Kollektiven auch thematisiert werden, bspw. als punktuelle Austauschsysteme oder schlicht als Interaktionen. Sie werden so aber gerade nicht als Kollektivhandeln analysiert. Ein Vergleich zwischen neuartigen und klassischen Formen in Bezug zu ihrer kollektiven Handlungsfähigkeit wird so also nicht möglich, da Handlungsfähigkeit in der colemanschen Konzeption stets einen vertraglich stabilisierten Handlungszusammenhang voraussetzt.

Jenseits dieses Grundproblems lassen sich drei weitere Grundannahmen hinterfragen, die das Konzept des Kollektivhandelns als korporativer Akteur implizieren:

(i) Das Konzept fokussiert auf vollständige Akteure und operiert mit einer harten Trennung: komplett Akteur zu sein oder gar nicht. Falls ein Kollektivakteur Akteur ist, ist er mit Potentialen zur interessierten Steuerung und Kontrolle von Situationen ausgestattet. Sein Vorbild für den Kollektivakteur ist dabei das freiheitlich-rational agierende Individuum der Aufklärung. Diese Annahmen vermitteln ein recht voraussetzungsvolles und zugleich reduktionistisches Verständnis des Akteurs. ${ }^{54}$ In Bezug auf das

54 Auf individueller kann man sich schon Ebene Situationen vorstellen, in denen Kontrolle und Steuerung zumindest eingeschränkt sind. Giddens (1984: 53) plädiert etwa unter Aufnahme der Entwicklungspsychologie Eriksons dafür, die grundlegende Differenz zwischen dem Handeln gesellschaftlich kompetenter Akteure und dem körperlichen Bewegen in ein Kontinuum zu überführen. Je nachdem, wie weit die ,transformation of the body into an instrument of acting- 
Kollektivhandeln werden somit nur spezifische Ordnungen und Sozialsysteme diskutiert, insbesondere die formal-hierarchische, zentral gesteuerte Organisation, die diesem Verständnis kompletter Akteure nahe kommt.

(ii) Damit verbunden ist auch ein ganz grundlegendes Problem der Handlungstheorie angesprochen, die kausale Verknüpfung von Akteur und Handeln. In der Handlungsphilosophie geht man seit langem von einer bedeutsamen Verschiebung in verschiedenen Denktraditionen aus, die man wie folgt zusammenfassen kann:

„[...] we shall examine and reject a view traditionally associated with the concept of agent causation, namely, that agents cause their actions; in our view, agents cause the results of their actions“"(Alvarez/Hyman 1998: 220).

Die in der colemanschen Fassung bereits vorausgesetzte Verbindung von Handeln und Interessen wird damit in ihrer prinzipiellen Verknüpfung infrage gestellt. Ob Kollektivakteure in ihrem Tun Resultate erzeugen können, die ihre Interessen verwirklichen, hängt schließlich von situativen Dynamiken ab, die nie vollständig kontrolliert werden können. Dies hat Coleman zwar stets betont, aber nie konsequent in seine Konzeption des Verhältnisses von Akteur und Handeln einbezogen. Bei ihm sind es stets die Akteure, die ein handeln erzeugen. Verschiedene Handlungskonzepte haben etwa, anders als Coleman, versucht, das Handeln nicht vorab als intentional oder kontrolliert Entwürfen folgend zu qualifizieren, sondern vielmehr verschiedene Grade der Intentionalität des Handelns unterschieden. So wird die Interessiertheit und Intentionalität des Handelns zu einer empirischen Frage. Diese ist bei Coleman bereits konzeptionell vorentschieden.

(iii) Bei Coleman ist ein Äquivalent zum weberschen subjektiv gemeinten Sinn für die kollektive Ebene alles andere als klar umrissen. Sicherlich kann ein solches in der Unterordnung unter ein kollektives Interesse gesehen werden, das eine intersubjektiv geteilte, sinnhafte Orientierung der Beteiligten darstellt. Zugleich spitzt Coleman mit einer derartigen Fokussierung auf ein kollektives Handeln als zweckrationales Handeln zu, da die gemeinsame Handlungsorientierung stets auf die Verwirklichung dieses Interesses bezo- 
gen bleibt. Nimmt man die Bandbreite der weberschen Handlungstypologie auf, so bleibt zumindest zu fragen, ob eine solche Zuspitzung auf das kollektiv-zweckrationale Handeln gerechtfertigt ist. Die empirische Frage dahinter ist, ob es nicht auch kollektiv-wertrationale, die (seit Durkheim thematisierten) kollektiv-affektuellen und/oder kollektiv-traditionellen Motive gibt. Die colemansche Übertragung steht ganz und gar im Zeichen der Zuspitzung auf das Handeln zweckrationaler Kollektivakteure, einer Zuspitzung, die keineswegs aus den grundlegenden Prämissen der Handlungstheorie Webers heraus gerechtfertigt ist.

Auch die Konstitution kollektiven Handelns hält einige Fallstricke bereit. Zunächst bedarf es, wie bereits angedeutet, einiger Arbeit, seine Ausführungen zum Kollektivhandeln auf das Makro-Mikro-Makro-Modell zu übertragen. Für das Modell scheint mir eine Spezifizierung in Bezug auf das Kollektivhandeln, vor allem in Bezug auf die Aggregation, zu fehlen. Hier zeigt sich der Ansatz erstaunlich stumm. Dies überrascht durchaus, stünden, bspw. im großen Vorbild der Korporation (dem weberschen Bürokratiemodell) doch mit Begriffen wie der Formalisierung und Prozeduralisierung von Handlungsabläufen durchaus Ansätze zur Verfügung. Weitere Problemkomplexe lassen sich vielmehr in dreifacher Weise zusammenfassen:

(i) Wenn auf einen einheitlichen Kollektivakteur fokussiert wird, dann geht dies bei Coleman mit einer Fokussierung auf eine bereits vorhandene, verbindliche und stabile Vertragsordnung einher. Dies impliziert eine Stabilität, die ob der eingangs beschriebenen Tendenzen zu situativen, temporären und fluiden Kollektiven zumindest zur Disposition zu stehen scheint. ${ }^{55}$ Auch

55 Dies liegt in der handlungstheoretischen Tradition auch darin begründet, wie kollektives Handeln dem Begriff des kollektiven Verhaltens gegenübergestellt wird, wie etwa aktuell bei Dolata und Schrape (2014: 19): „Während sich nicht-organisiertes kollektives Verhalten auf der Grundlage von allgemein verfügbaren Infrastrukturen des Kollektiven entwickelt, ist für kollektive Akteure und kollektives Handeln also eine sukzessive Institutionalisierung des Kollektiven typisch, die in eigenständigen Organisierungs- und Strukturierungsleistungen der Gemeinschaft oder der Bewegung ihren Ausdruck findet." Die Autoren beschreiben hierbei einen harten Übergang zwischen unorganisierten Kollektiven, die sich kollektiv verhalten, und Kollektivakteuren, die in der Lage sind, strukturiert kollektiv zu handeln. Diesen Übergang beschreiben sie als einen zwischen Akteur-Aggregaten, die mit keiner eigenen Strategiefähigkeit ausgestattet sind, und sogenannten ,komplexen Akteuren [...] bei denen die ,Intention“ intenti- 
wenn die vertragliche Ordnung nicht im formal-juristischen Sinne gedacht wird, ist dennoch fraglich, ob eine gemeinsam ausgehandelte Ordnung, die bspw. Rechte, Pflichten und Rollenerwartungen in der Mitgliedschaft beinhaltet, noch die einzig denkbare Form der Kooperation ist. ${ }^{56}$

(ii) Das vertragstheoretische Argument impliziert vor allem, dass es sich im Kollektivhandeln um eine Verbindung von Legitimitäts-, Belohnungs- und Sanktionierungsmustern mit einem Herrschaftsthema handelt. Die kollektive Prägung von Mustern der Situationsdeutung oder dessen, was legitimes Verhalten im Sinne der Organisation ist, wird vertraglich ausgestaltet. Dies unterschlägt die Rolle einer gemeinsamen Vergangenheit und gemeinsamer Erfahrungen für eine kollektive Sinnstiftung, für die es in der Organisationsforschung zahlreiche Belege gibt (vgl. Weick et al. 2005).

(iii) Aus forschungspragmatischen Gründen werden Korporationen konzeptionell mit Individuen gleichgesetzt (Coleman 1992: 117), wie es auch das amerikanische Recht mitunter vorsieht. Die Aufnahme der kollektiven Rechtspersonen in den Sozialwissenschaften reicht zurück bis zu Weber (1972: 6f.). Das Argument, dass die rechtliche Formalisierung eine solche Gleichbehandlung kennt, macht aber diese Gleichsetzung nicht weniger problematisch. ${ }^{57}$ Wie bereits beschrieben wird der Übergang vom Individuellen zum Kollektiven in der konzeptionellen Übertragung des Akteursbegriffs durchaus thematisiert. Ein bedeutsamer Aspekt fehlt dabei jedoch: Sowohl in der Selektion als auch in der Aufnahme der gesellschaftlichen Situation sind kollektive Akteure, kollektive Interessen und ihre vertragliche

onalen Handelns sich auf die von den beteiligten Individuen erwartete gemeinsame Wirkung bezieht" (Scharpf 2000: 101). Zusammengenommen und zugespitzt kann man also formulieren, dass kollektives Handeln hier eines ist, das geteilte Absichten und eigene Organisierungsweisen im Kollektiv voraussetzt. Man muss folglich von einem institutionalisierten Kollektiv ausgehen, wenn man kollektives Handeln so voraussetzungsvoll an Kollektivinteressen oder intentionen bindet.

56 Siehe die Literatur zu partiellen Organisationen (Ahrne/Brunsson 2011) oder heterogener Kooperation (Strübing et al. 2004) für Gegenbeispiele.

57 Von zentraler Bedeutung ist die damit einhergehende Setzung, dass auch ein Kollektivinteresse Interesse eines Akteurs ist. Natürliche Personen und Korporationen können in modernen Gesellschaften beide als Akteure auftreten (Coleman 1990: 542), was für Coleman bedeutet, Ressourcen zu kontrollieren, Interessen auszubilden und zu vertreten sowie Rechte und Verantwortlichkeiten wahrzunehmen. Das heißt auch: Korporationen können in bestimmten Situationen als funktionale Äquivalente von natürlichen Personen gelten (ebd.: 537), die Akteursfunktionen wahrnehmen. 
Basis abhängig von den Lesarten und Interpretationen der beteiligten Individuen. Hierauf hat Giddens schon früh hingewiesen:

„[...] It is perfectly true that a corporation can be an agent in law. But laws have to be interpreted, and applied; it takes human agents to do that, as well as to frame them in the first place. [...] Thus they say that, if we impute any universal attributes to human subjects, it follows that social relations 'are relations between subjects and they exist in and through the will and consciousness of subjects' (p. 268). But it does not follow at all; although certainly no approach which ignores the will and consciousness of human subjects is likely to be of much use in social theory“ (Giddens 1979: 272, Herv. RJ).

Dieser giddenssche Impetus auf die subjektive Konstitution müsste hierbei durchaus im Sinne des methodologischen Individualismus sein. In fundamentaler Weise sind auch über Mitgliedschaftsrollen definierte und stabilisierte Kontexte kollektiven Handelns an subjektive Interpretationen und Aktualisierungen gebunden. Mit Weber gesprochen, erhöhen Korporationen durch ihren ausgefeilten Herrschaftsapparat nur die Chance, auf einen Befehl im Sinne des Korporationsinteresses bei ihren Mitgliedern auch ein Handeln zu erzeugen, sie determinieren diesen Vorgang damit jedoch keineswegs. Hier offenbaren sich interne Inkonsistenzen des Ansatzes. ${ }^{58}$

(iv) Der sicher grundlegendste Einwand gegen das präsentierte Modell ist der der einseitigen Fokussierung und Naturalisierung der Mikroebene im Konstitutionsverständnis. Beides geht mit dem Makro-Mikro- Makro-Modell der Erklärung einher. Im Kollektivhandeln wird etwa die Verbindlichkeit einer vertraglichen Ordnung für die Erzeugung eines intendierten, kollektiven Outputs über die Mikroebene erklärt. Dabei stellt sich zunächst erst einmal die Frage, ob nicht gesamtgesellschaftlich oder zumindest übergreifende Dynamiken ins Feld zu führen wären. Nehmen wir die Zusammenarbeit im Betrieb als ein Beispiel kollektiven Handelns, so würde etwa Marx (1969: 183ff.) den „doppelt freien Lohnarbeiter“ als eine umfassende gesellschaftliche Voraussetzung für den Betrieb definieren. Bereits Weber (1979) hat zudem darauf hingewiesen, dass gesellschaftlicher Rationalisie-

58 Die von Abraham und Büschges (2004) eingeführte, doppelte Badewanne, die versucht, auf dieses Problem zu reagieren, verweist ebenfalls auf die eigene Entscheidungsfähigkeit der Korporationen. Sie zieht in der Badewanne noch eine Meso-Ebene der korporativen Akteure ein. Dies verdeutlicht das ganze Problem allerdings anstatt es zu lösen. In der Logik des Ansatzes sind auch kollektive Interessen ganz basal in den Wahlhandlungen der Individuen begründet. Die Gleich All dies konterkariert die prinzipielle Mikrofundierung des Ansatzes. 
rungstendenzen für den modernen kapitalistischen Betrieb unabdingbar sind. Sicher gibt es mit den erwähnten, gesellschaftstheoretischen Schriften Colemans Ansatzpunkte eines Einbezugs derartiger gesamtgesellschaftlicher Dynamiken. Im Zentrum des Konzeptes kollektiven Handelns steht jedoch deutlich die Verbindlichkeit einer Vertragsordnung für das individuelle Handeln. Der Blick darauf, wie gerade aus einem Arrangement verschiedener Ordnungen Kollektivhandeln erwächst, wird somit zumindest dezentriert.

Die Mikroebene bildet weiterhin eine Art natürliche Basis des Erklärens. In Bezug auf das Kollektivhandeln kommt etwa der Figur der Ressourcenzusammenlegung eine zentrale Bedeutung zu. Hierbei werden die Ressourcen als Besitztümer der Akteure betrachtet, über die sie frei verfügen und die sie daher einbringen bzw. investieren können. Fragen danach, worauf diese freie Verfügung beruht, stellt sich in dieser Theorierichtung zunächst nicht. Auch der Legitimationsaspekt der Ressourcenverwendung wird in diesem Zusammenhang vernachlässigt. ${ }^{59}$

59 Auch woher etwa die Situationsdeutungen oder Handlungsrahmen kommen, die zur Situationsaufnahme führen, ebenso wie die Präferenzen, an denen sich die Selektion orientiert, werden innerhalb des Theorieansatzes wiederrum ,ausgelagert“. Hier wird die Theorie mit Annahmen der normativen Integration à la Parsons (wie bei Coleman) oder neuerdings der praxistheoretisch thematisierten Bedeutung von implizitem Wissen und Routinen erweitert (siehe SchulzSchaeffer 2010). Die individuelle Basis kann also mit verschiedenem Bestand der Soziologie spezifiziert werden (das Herausstellen dieser flexiblen „Bestückung“ ist ein genuines Verdienst der Weiterführung des colemanschen Programms bei Esser 1999) und ist an sich zur Erklärung ausreichend. Die Frage, warum diese Basis noch so bedeutsam ist, kann man sich ob dieser Bewegung aber stellen. Dennoch hat die Naturalisierung der Mikroebene aufgrund dieser Tendenzen an ihrer Problematik nichts eingebüßt, da man sie letztlich noch immer als Letztelement behandelt, auf das man nur alle Ergebnisse hin beziehen muss, um sie zu erklären. Die Frage, ob die Soziologie ein solches Letztelement angeben kann, ist noch immer heftig umstritten. Sicher kann man ob solcher Beharrlichkeit verzweifelt fragen: „Wie oft noch, wie lange noch?“ (Esser 2000). Die Antwort ist denkbar einfach: solange Formalisierungsbemühungen und die gesellschaftliche Plausibilität der Naturalisierung des Individuums allein noch keine hinreichenden Begründungen für grundlegende Theorieentscheidungen sind. 
2.2 Kollektivhandeln, Skripte kollektiver Akteure und die Moderne

(A) Kollektivhandeln als institutionalisiertes Skript und Handeln einer Akteursidentität

Bedeutend für ein institutionalistisches Verständnis kollektiven Handelns ist schon bei Durkheim, dass zur kausalen Intervention fähige Einheiten und Formen des Ausführens aus interinstitutionellen Dynamiken entstehen. Es geht um gesellschaftlich anerkannte, legitimierte und vorausgesetzte Typisierungen kollektiver Akteure und kollektiver Handlungsfähigkeit (siehe I.1.2.). Diesem Argument folgt auch der World-Polity-Ansatz. Durch die Aufklärung in der westlichen Welt führen Individuen nicht mehr nur einen göttlichen Plan aus, sondern beginnen, die Welt eigenständig zu verändern (Meyer et al. 1987). Die interessante Frage ist dann, mit welchen Eigenschaften, insbesondere welchen Machtbefugnissen, Verantwortlichkeiten und Handlungskapazitäten typisierte Akteure dabei gesellschaftlich ausgestattet werden (Meyer/Jepperson 2000: 106).

Aufgenommen wird diese Frage mit dem Begriff der Agency. Dieser bezeichnet das legitime Ausführen von Aktivitäten, die als Repräsentation eines (ebenfalls legitimen) Prinzipals gelten:

\footnotetext{
„By ,agency’ we refer to legitimated representation of some legitimated principal, which may be an individual, an actual or potential organization, a nation-state, or abstract principles (like those of law or science, or more prosaically, high culture or even etiquette). Note that the concept ,agency" directly draws attention to the devolution of external authority, and to the external legitimation and chartering of activity" (ebd.: 101).
}

Hierbei rekurrieren die Autoren (ebd.) auf der (auch bei Coleman zu findenden) Trennung eines Prinzipals im Sinne einer Handeln „beauftragenden“ Entität und eines Agenten, der das Handeln tatsächlich ausführt. Sie wenden diese Bestimmung aber sogleich konstruktivistisch: Es geht um gesellschaftliche Institutionalisierungsprozesse, die bestimmten Akteuren in institutionell definierten Bereichen ein Intervenieren in die Welt hinein als legitimes Ausführen eines legitimen Handlungsprinzips ermöglichen (wie beschränken). Die Autoren unterscheiden hierbei vier Typen von Prinzipien: das Handeln im Sinne des Selbst, abstrakter Prinzipien, anderer Akteure oder nicht-akteurhafter Einheiten (ebd.: 106ff.). Bedeutsam ist folglich die Zuschreibung des Repräsentierens legitimer Prinzipien. Agency muss sich dabei als legitime Repräsentation eines bestimmten Prinzips darstellen können. Mit kollektiver Agency haben wir es meines Erachtens folglich immer dann zu tun, wenn es sich beim repräsentierten Prinzip um 
das Selbst eines Kollektivakteurs (hier: einer Organisation bzw. eines Staates) oder eines kollektiv geltenden Prinzips (etwa den Weltfrieden) handelt.

Dieses legitime Intervenieren kann sowohl vom Akteur als auch vom Handeln getrennt werden, ${ }^{60}$ alle drei speisen sich aus der institutionellen Umwelt:

„In phenomenological models, the institutional environment operates more as a cultural or meaning system, penetrating actors far beyond their boundaries and constructing agency, identity, and activity. [...] the actor on the social stage is a scripted identity and enacts scripted action" (Meyer 2010: 4).

Der Begriff des Kollektivakteurs, der hier für Organisationen und Nationalstaaten reserviert wird, meint also zunächst nicht mehr als eine gesellschaftlich als Akteur geskriptete Identität. Kollektives Handeln bezeichnet geskriptete Handlungsformen, die gesellschaftlich als kollektiv verstanden werden. So folgt die Identität eines Staates ebenso gesellschaftlichen Skripten, wie mit ihnen typische Aktivitäten, etwa die Grenzsicherung oder das Gewaltmonopol, gesellschaftlich verbunden werden. Im Resultat zeigen sich enorme Ähnlichkeiten zwischen den real existierenden Staaten. Was macht beides aber nun kollektiv? Als kollektives Handeln bezeichnet man Aktivitäten, die gesellschaftich anerkannten Skripten des Kollektivhandelns folgen und somit als kollektiv (und nicht individuell) anerkannt werden. Das mündet in eine tautologische Definition. Spezifischer werden die Autoren in Bezug auf die Akteure. Gesellschaftlich wird drei Typen von Akteuren eigenständige Handlungsfähigkeit zugeschrieben. Staaten und Organisationen als Kollektivakteure werden dabei von Individuen unterschieden. ${ }^{61} \mathrm{Sie}$ sind mit einer eigenen Actorhood ausgestattet. Für Organisationen bedeutet dies bspw.:

60 Vor allem die neueren Arbeiten von John W. Meyer $(2008,2010)$ legen dies nahe, und auch in Meyer/Jepperson (2000) lassen sich Hinweise darauf finden. Ich danke Arnold Windeler für diesen Hinweis. Nichtsdestoweniger kann man sicher auch für eine Gleichsetzung von Agency und Handeln plädieren, auch dazu lassen sich Hinweise finden. Die Textgrundlage ist an diesem Punkt keineswegs klar. Der Dreischritt nimmt meines Erachtens aber deutlicher auf, dass es neben der Paketierung aus Akteur und Handlung auch noch weitere legitime Repräsentationsformen gibt, etwa umfassendere Prinzipien oder ein Sich-Einsetzen für die Wale.

61 Neuerdings werden dabei zumindest einige Spezifika von Organisationen als Akteurstyp diskutiert (Bromley/Meyer 2015: 128ff. sowie 141ff.). Eine systematische Abgrenzung und vergleichende Diskussion der Typen erfolgt meines Wissens nicht. 
„[...] organizations have sovereign accountability in legal and moral terms, and they are expected to define their own boundaries, purposes, and responsibilities" (Bromley/Meyer 2015: $3)$.

Der Status als Akteur geht also mit bestimmten gesellschaftlichen Erwartungen einher. Die Akteursidentitäten von Organisationen und Staaten sind etwa mit typischen Machtbefugnissen, Rechten und Pflichten sowie Kompetenzen und Fähigkeitsannahmen verbunden (Meyer/Jepperson 2000: 106). ${ }^{62}$ All das wirkt sich ganz entscheidend auf die Agency und das Handeln von Kollektiven aus. Die Autoren fokussieren hier vor allem Wege, die Anforderungen zu erfüllen, die der Akteursstatus mit sich bringt.

\section{Die Konstitution kollektiven Handelns über interinstitutionelle} Dynamiken

Der World-Polity-Ansatz ist in der Thematisierung der Entstehung, Aufrechterhaltung und Veränderung kollektiven Handelns durch eine starke Betonung des klassischen institutionalistischen Programms in der Tradition Durkheims gekennzeichnet. Die Konstitution moderner Sozialität und von Kollektivakteuren ist hierbei zutiefst geprägt durch „Polity-Formen“ im doppelten Wortsinne, also eines tatsächlichen Gemeinwesens und dieses Gemeinwesen tragende Manieren und Taktformen, d.h. institutionalisierter Regeln. Die institutionellen Formen der Agency, des Handelns und die mit einer Actorhood ausgestatteten Akteursidentitäten speisen sich aus Institutionalisierungsprozessen, die die Autoren gesellschaftstheoretisch unter Verweis auf Webers Rationalisierungsthese bestimmen (vgl. Meyer et al. 1987). Gleichzeitig, und das kennzeichnet ein grundlegendes Spannungsverhältnis in diesem Ansatz, wird immer wieder auf eine handlungstheoretisch-phänomenologische Fundierung der Institutionen im Sinne Berger und Luckmanns (1980) verwiesen. Die Autoren fassen die Regelmäßigkeiten des modernen Soziallebens als Ergebnis des Institutionalisierungsprozesse kenn-

62 Frank Meier (2009: 85ff.) hat diese Implikationen der Konstruktion als Akteur im Sinne des grundlegenden Aufsatzes von Meyer und Jepperson (2000) weiter spezifiziert und unterscheidet zwischen dem Recht und der Verantwortlichkeit zur Ausprägung von Identität (was Einheit und Abgrenzung, Autonomie, sowie kategoriale Zuordnung und Besonderheit einschließt), Kompetenzen (was Handlungs- und Entscheidungsfähigkeit, Selbstkontrolle sowie Kognitionsfähigkeit beinhaltet) und Orientierungen (im Sinne von Handlungsorientierung und Absichten). 
zeichnenden Dreischritts aus Internalisierung, Legitimation und Objektivation. Der Ansatz fokussiert aber vor allem die interinstitutionelle Ebene. Er erklärt Soziales über ein spezifisches Bündel sich wechselseitig stützender Institutionen, die die Actorhood in der westlichen Moderne begründen. ${ }^{63}$

Die Homogenisierung der modernen Welt wird getragen von weltweit sich ausdehnenden institutionellen Regeln im Sinne umfassender Rationalisierung und Säkularisierung, die Actorhood zuweisen. Mit dieser sind wiederum Formen kollektiven Handelns verbunden. Diese Rationalisierung von Welt steht in einer „reciprocal relation to the social construction of the actors given ontological status in society" (Meyer et al. 1987: 25). Das meint insbesondere, dass im historischen Verlauf die Welt immer stärker nicht mehr als eine gottgegebene, sondern menschenbestimmte gedacht wird. Es geht also um die Übertragung legitimer Handlungsautoritäten von göttlichen Mächten in die Gesellschaft hinein (Meyer/Jepperson 2000: 101). Wir sehen hier die direkte Verwandtschaft zu Durkheim, denn es handelt sich um eine veränderte Klassifikation dessen, was als die Welt verändernder Faktor angesehen wird.

Was als soziales Handeln und wer als Handelnder gilt, ist damit aus dieser Perspektive nicht naturgegeben: Es ist selbst eine Konstruktionsleistung (Meyer 2010: 4). Hinter diesem konstruktivistischen Handlungs- und Akteursverständnis verbirgt sich die Einsicht in eine untrennbare Verbindung von Handeln und Handelndem:

,actorhood is a role or identity, as in a theatrical world (Frank and Meyer 2002): individual actors, in this usage, have socially conferred rights and responsibilities, and socially conferred agency to represent these (and other) interests (Meyer \& Jepperson, 2000). Actorhood [...] is scripted by institutional structures; and the relation between actor and action is no longer a simple causal one - both elements have institutional scripts behind them, and their relation has, causally speaking, strong elements of socially constructed tautology. That is, the actor-action relation is a package and as people and groups enter into particular forms of actorhood, the appropriate actions come along and are not usefully to be seen as choices and decisions" (Meyer 2008: 794, Herv. RJ).

Die institutionalisierten, wechselseitig typisierten Handlungs- und Akteursformen bilden ein einander stützendes Paket, aus dem heraus sowohl das Handeln

63 Diese haben ihre Wurzeln, und hier schließen die Autoren eher lose an Weber an und deuten ihn in eine spezifische Richtung aus, in der spezifisch westlichen Ausrichtung von Rationalisierungsprozessen an zwei Kollektivzielen. Für sie ist die westliche Moderne ein „rational project of creating progress and justice“" (Meyer et al. 1987: 25). 
als auch der Akteur verstanden werden muss. Gleiches trifft auch auf Kollektivhandeln und den Kollektivakteur zu. Beide sind nur im Paket zu bekommen. Ein Akteursstatus (im Sinne von Actorhood) wird durch typisierte Formen angemessenen Handelns gestützt. So überformen durchaus fest gebundene Pakete aus kollektivem Akteursstatus und adäquatem Handeln, die aus den beschriebenen interinstitutionellen Dynamiken entstehen, das Tun und die Interessen der ,rohen“ Akteure. Als institutionelle Pakete ermöglichen sie ein Handeln, das auch gesellschaftlich als kollektives Handeln und Handeln eines Kollektivakteurs verstanden wird. Die Fähigkeit zum legitimen Intervenieren (Agency) ${ }^{64}$ ergibt sich zudem aus der ebenfalls typisierten Möglichkeit zur Interessensrepräsentation. Akteure können ihr Tun auf ein legitimes Interesse, z.B. den Weltfrieden, beziehen. Auch hierfür sind die geskripteten Akteur-Handlungs-Pakete zentrale Bezugspunkte. Institutionalisierte Akteure können dabei sowohl legitim eigene Interessen vertreten, als auch zutiefst mit allgemeinen Prinzipien oder Interessen anderer verknüpft sein und daraus auch ihre Position als Akteur legitimieren. So basieren die Interventionsmöglichkeiten, Handlungsformate und Akteursidentität von Umweltschutzorganisationen zutiefst auf den Interessen anderer, etwa denen der Wale.

64 Die Agency-Figur des World-Polity-Ansatzes wurde jedoch kaum ausgearbeitet. Am ehesten lässt sie sich verstehen als kognitiv-normative Konstruktion des entschiedenen, expliziten und legitimen Eingreifens in Welt zur Veränderung dieser. Dieses Konstrukt umspannt in der Moderne immer weitere Bereiche und wird durch das wissenschaftliche Wissen um die Veränderungsmöglichkeiten gestützt: „Actor agency is made real through the highly expanded educational systems now found everywhere. These meld the principles of scientized knowledge into the selves of entitled persons, constructing empowered individual actors capable of building society through their choices. Much social structure, then, turns into modern formal organization, assembling individual actors into structures of mobilized participation. The individuals and organizations so created now with the standing of agentic actors, commonly act on behalf of the great principles that empower their agency. Far from ordinary self-interest, they often act as mobilized Others, creating expanded versions of actorhood" (Meyer 2010: 15). Das Bildungswesen pflanzt also die Möglichkeit zur Veränderung in unsere Köpfe und schreibt ebenso die Möglichkeit zur organisierten Intervention in Welt in uns ein. So entstehen im Verbund mit einem legitimen Akteursstatus tatsächlich wirksame Organisationen und Individuen, die real wirksames, kollektiv mobilisiertes Tun aktualisieren und aktualisieren können. Im Gegensatz zu den Handlungstheoretikern agieren sie aber in der Regel gerade nicht im Sinne des Objektselbst, eines kollektiven oder individuellen Selbstinteresses. Dies reicht in modernen Gesellschaften nicht mehr aus. Vielmehr sind sie zumeist Agenten für weitaus umfassendere Prinzipale, also allgemeine Prinzipien wie eben der Gleichheit und Gerechtigkeit. Sie treiben damit als Handlungsmaterial einen Prozess voran, dessen Produkt ihre Agency, ihre Aktivitäten und ihr Akteursstatus selbst erst ist: die spezifisch gerichtete, westliche Rationalisierung (Meyer et al. 1987). 
(C) Die Institutionalisierung, Legitimation und gesellschaftliche Zuschreibung kollektiven Handelns

Die zugespitzten und häufig im besten Sinne kontraintuitiven Ausführungen des World-Polity-Ansatzes sind eine bedeutsame Gegenbewegung zur Naturalisierung von Handlungsfähigkeit in der handlungstheoretischen Tradition oder in zahlreichen empirischen Studien. Sie betten die Diskussion um kollektive Handlungsfähigkeit in einen umfassenden, gesellschaftstheoretischen Kontext ein, plädieren aber auch sozialtheoretisch für eine konstruktivistische Betrachtung. Sie plädieren also für eine Dekonstruktion und kulturspezifische Rekonstruktion von sozial anerkannten Formen legitimen Intervenierens in der Welt, des Handelns sowie des Akteur-Seins und einem Fokussieren der Anforderungen, die diese Sozialformen mit sich bringen. Damit eröffnet der World-Polity-Ansatz uns nicht nur eine interessante Fragerichtung nach den gesellschaftlichen Quellen kollektiver Handlungsfähigkeit, sondern hilft uns mit dieser Fragestellung auch typische Inkonsistenzen in diesen Anforderungen an Kollektive zu verstehen (siehe für Organisationen z.B. Bromley/Meyer 2015: 149ff.). Auch weisen klassische Konzepte wie das der Entkopplung oder des Fassadenbildens (Meyer/Rowan 1977) auf Möglichkeiten hin, wie die Spannungen zwischen „rohen“ und ,gesellschaftlich geskripteten“ Formen in ihren praktischen Relationen zu fassen sind. ${ }^{65}$

In Bezug auf die Konstitution kollektiven Handelns kommt dem alten Thema der Legitimität und ihrer homogenisierenden Wirkung wieder stärkere Beachtung zu. Dieses wird mit dem seit Durkheim für die Soziologie so bedeutsamen Aufdecken und Hinterfragen gesellschaftlicher Wahrnehmungs-, Interpretations- und Klassifikationsweisen verbunden. Eine gesellschaftlich geteilte und umfassende Weltsicht umfasst auch die für handlungsfähig gehaltenen Kollektive. Es geht darum, dass es zunächst von grundlegender Bedeutung ist, dass Kollektivhandeln als solches interpretiert, klassifiziert und wahrgenommen wird. Diesen gesellschaftlichen Zuschreibungsprozesse von Akteur-Sein, Aktivitäten sowie Interventionspotentialen weist der Ansatz eine hohe Bedeutung für die Konstitution zu. Dieser Punkt Durkheims wird hierbei in konkrete Skripte über-

65 Auch dieses klassische Thema Bedarf jedoch noch weiterer konzeptioneller Anstrengungen. Siehe etwa die differenzierte Aufnahme bei Kirchner und Meyer (2016) für einen fruchtbaren Weg. 
setzt und zumindest in Bezug auf organisationale Kollektivakteure operationalisiert (siehe Meier 2009, Bromley/Meyer 2015).

Zudem wird neben den beschriebenen, umfassenden gesellschaftlichen Quellen Webers alte Idee der intermediären Einrichtungen des rationalisierten Rechts, des Rechnungswesens sowie der Professionen wiederbelebt. Somit wird auch sozialen Ordnungen der Mesoebene jenseits des Staates und der Organisation eine zentrale Bedeutung für die Angleichungstendenzen im Kollektivhandeln zugesprochen und eine Mehrebenenbetrachtung der Konstitution kollektiven Handelns eingeführt.

(D) Theoretische Herausforderungen eines institutionalistischen Zugriffs

Allgemein kann der eingangs thematisierte Kritikpunkt der Akteursfixierung auch für den soziologischen Neo-Institutionalismus in Anschlag gebracht werden. Akteure sind auch in der World Polity von zentraler Bedeutung in der Konzeption kollektiven Handelns. Die Autoren schreiben so die von ihnen beobachtete, spezifisch westlich-moderne Fixierung auf den Akteur auch in den Sozialwissenschaften selbst fort (vgl. Meyer/Jepperson 2000: 100). ${ }^{66}$ Dies ist keineswegs unproblematisch, will man die eingangs beschriebenen Tendenzen situativer, temporärer und fluider Kollektivität in den Blick nehmen. ${ }^{67}$ Letztlich thematisiert der Ansatz, dass bislang nur Organisationen und Nationalstaaten gesellschaftlich Handlungsfähigkeit zugesprochen wird. In der Logik institutionellen Denkens wäre zunächst kritisch zu diskutieren, on die Gesellschaft selbst in ihren Typisierungen von Akteuren hier nicht schon deutlich differenzierter ist, eine breitere Palette an institutionalisierten Akteuren kennt. Neben diesem Problem ergeben sich jedoch zwei weitere, ungeklärte Fragen in Bezug auf das Konzept des Kollektivhandelns:

66 Auch und gerade die Neo-Institutionalistische Tradition schreibt diese Geschichte der Akteursfixierung fort. Zwar entlarvt sie die dahinterliegende, kulturelle Konstruktion, gleichzeitig bindet sie aber die Fähigkeit zur Ausübung von Agency an Akteurspositionen, behandelt beide als zutiefst miteinander verwobene und einander stützende „Pakete“"(siehe I.2).

67 Sicher gibt es andere Ansätze hierfür innerhalb der breiteren neo-institutionalistischen Literatur jenseits der World Polity (siehe früh etwa Brunsson/Sahlin-Andersson 2000 für die skandinavische Tradition). 
(i) Eine besonders schwerwiegende Leerstelle ist die fehlende Differenzierung zwischen individuellem und kollektivem Handeln. Hier hat die Theorie selbst nicht mehr zu sagen, als dass es um gesellschaftlich als kollektiv zugeschriebenes Handeln geht. Kollektivakteure werden mit denselben begrifflichen Mitteln analysiert wie Individuen, denn sie alle müssen als moderne Skriptung des Akteurs und des Handelns, ,as taking up standardized technologies of agentic authority" (Meyer/Jepperson 2000: 117), behandelt werden. Sie nehmen dann eben spezifisch für Organisationen geltende, standardisierte und als Strukturen der mobilisierten Partizipation von Individuen angesehene Technologien der Handlungsautorität auf. ${ }^{68}$ Das ist sicher zunächst eine große Stärke des Ansatzes. Weder Individuen noch Kollektive werden mit einer quasi natürlichen Handlungsfähigkeit ausgestattet. Organisationen werden meist als geprägt durch kulturell-legitime Modelle des Organisierens (vgl. z.B. Dobbin 1994) betrachtet. Es kommt aber lediglich zu Andeutungen dessen, worin sich individuelle und kollektive Skripte unterscheiden. $\mathrm{Zu}$ klären wäre, ob neben aller Homogenität auch Unterschiede in den Konstruktionen vorherrschen. Das ist insbesondere eine empirische Frage. Auch die Beziehung zwischen Skripten auf individueller und kollektiver Ebene wird nur mit Andeutungen abgefunden. In beiden Punkten zeigt sich der World-Polity-Ansatz bislang reichlich stumm. ${ }^{69}$ Diese Tendenzen hängen aber auch mit der engen Verbindung des Ansatzes mit der gesellschaftstheoretischen These der Homogenisierung der westlichen Welt zusammen.

68 Für das Kollektivhandeln von Organisationen müssten folglich auch spezifische (Kollektiv-) Handlungsskripte zur Verfügung stehen. Pedersen und Dobbin (1997: 439ff.) zeigen bspw. in einer historischen Analyse für die USA auf, wie die rechtliche Übernahme der europäischen Klassifikation durch die Suche nach Ähnlichkeiten von Organisationen als rechtskräftigen Korporativakteuren nicht nur zur rasanten Verbreitung dieser im 19. Jahrhundert führte, sondern auch Transaktionen zwischen ihnen wie bspw. Landkauf etc. erst ermöglichte. Diese Skripte würden spezifische Formen des Handelns zwischen Organisationen hervorrufen, tatsächlich wirksames Tun zwischen den Organisationsakteuren zur Folge haben. Ein systematisches Weiterverfolgen einer eigenständigen Organisationstheorie steht trotz dieser Ansätze weiterhin aus (vgl. King et al. 2010, Kirchner et al. 2015), von einer allgemeinen Differenzierung individueller und kollektiver Akteure ganz zu schweigen.

69 Vgl. zu einem neueren Versuch der Spezifizierung in Bezug auf Individuum und Organisation Bromley/Meyer 2015: 125ff., die allerdings gerade die Beziehung zwischen beiden nicht thematisieren. 
(ii) Weiterhin ist das Handlungsverständnis nur unzureichend geklärt. Begriffe wie Aktivitäten, Praktiken und Handlungen, die in der Sozialtheorie üblicherweise Verschiedenes meinen, werden hier synonym verwendet. Schon früh wurde Handeln hierbei unter Verweis auf Berger und Luckmann (1980) als sozial konstruiert, musterhaft und durch gesellschaftliche Institutionen geprägt beschrieben (Meyer et al. 1987: 22). Es wurde sich bislang mit der Bemerkung abgefunden, dass das, was Akteure tun, der gesellschaftlichen Definition des Akteurs bereits inhärent ist (ebd.). Die durchaus mit den Anleihen an die phänomenologische Tradition mögliche Gleichberechtigung des Themas sozialkonstruierten Handelns und Akteur-Seins wird dann zu Gunsten des Akteur-Seins entschieden, eine Diskussion von Handlungsskripten früh aufgegeben. Auch die Beziehung zwischen Handeln und Akteur, ebenso wie das zwischen Handeln und Agency kann in den Texten verschiedentlich interpretiert, keineswegs aber als dezidiert geklärt beschrieben werden.

Auch in Bezug auf Konstitutionsfragen wurden am Neo-Institutionalismus klassische Kritiken geäußert, die mit den Problemlinien der mangelnden Aufnahme von Heterogenität und Diversität, der Flexibilität und des Wandels sowie der Unterbetonung von Konflikten ${ }^{70}$ auf Formen kollektiven Handelns bezogen werden können (siehe klassisch DiMaggio 1988, Powell 1991, Hofmann 1999, für einen Überblick Kirchner et al. 2015). Insgesamt erweist sich der Ansatz als mit einer zutiefst strukturalistischen Grundfigur ausgestattet, die den interinstitutionellen Dynamiken nach der Entzauberung der Welt die Akteursidentitäten, Handlungs- und Agencyformen als machtvolle Mittlerinstanzen an die Hand gibt, Institutionen letztlich aber dennoch eine die konkreten Aktivitäten bestimmende Rolle zuweist. Diese allgemeinen Zuspitzungen gehen mit drei weiteren Herausforderungen für die Zwecke des in der Einleitung skizzierten analytischen Zugriffs auf situative, temporäre und fluide Formen von Kollektivität einher:

70 Der Neo-Institutionalismus leugnet Konflikte keineswegs (vgl. Koenig/Dierkes 2011). Es geht hier vielmehr darum, dass diesen Konflikten vorgeordnete Prozesse der Wahrnehmung und Interpretation von Welt eine wesentlich größere Bedeutung zukommt. Die Alternativen, um die Konflikte sich ranken, entspringen bereits einem stark zugerichteten Ausschnitt von Welt. 
(i) Der Fokus auf bereits institutionalisierte und einander stützende, kollektive Akteur-Handlungs-Pakete impliziert eine Konzentration auf hochgradig stabile Kontexte des Kollektiven. Nicht umsonst existieren nur zwei Typen kollektiver Akteure: Organisationen und Nationalstaaten.

(ii) Auf die Bedeutung des Subjekts für die Konstitution angesprochen, erfolgt zumeist ein Verweis auf die Phänomenologie. Bei den Skripten handelt es sich um in Sozialisationsprozessen vermittelte Institutionalisierungsprozesse. Das Verhältnis von Skriptungen und alltäglichem Handeln ist damit aber keineswegs geklärt, ebenso wie die Rolle des Subjekts. In den Texten deuten viele Formulierungen auf eine Regelanwendung hin. Hierbei haben viele Autoren herausgearbeitet, dass jede Regelanwendung stets eine Regelwendung bedeutet (siehe z.B. Ortmann 1995). Für Prozesse der situativinteraktiven Interpretation und graduellen Abwandlung von Skripten im Handeln, die auch Wandel erklärbar machen könnten, liegt in dieser Tradition kein Konzept vor. ${ }^{71}$ Die Kompetenzen, Positionen oder Ressourcen, die nötig sind, um stabile Skripte zu erschaffen bzw. umzudeuten, sind allesamt Leerstellen in der Theorie. Dasselbe gilt für die Rahmung der Situation, die Agierende erkennen lässt, welche Skripte denn nun anzulegen sind.

(iii) Auch der aus Goffmans dramaturgischer Soziologie entlehnte Skriptbegriff selbst wird nur metaphorisch aufgenommen. Hierbei verschenkt der Ansatz bedeutsame Potentiale einer interaktionsbezogenen Fundierung. Diese könnte nicht nur das Forschungsprogramm an empirisch-qualitative Forschungen anschlussfähiger machen, sondern auch und vor allem helfen, die Skriptungsidee konzeptionell ernst zu nehmen. ${ }^{72}$

71 Siehe bspw. Goldenstein und Walgenbach (2016) für diese Kritik und einen Vorschlag in Bezug auf die subjektive Konstitution einer gemeinsamen Praxis.

72 Steve Barley (2008) beschreibt diese Anschlüsse als „Coal Face Institutionalism” und hat seit den 1980er Jahren eine ganze Reihe an Vorschlägen zu einem interaktionsbezogenen Verständnis von Skripten gemacht und diese in einer Vielzahl an Anwendungsfällen konkret werden lassen (Barley 1986, 1989, Barley/Tolbert 1997). Er spezifiziert hierbei Skripte ganz im Sinne Goffmans: „A script organizes and typifies interaction by defining how parties to an encounter should play their roles" (Barley 2015: 35). Die Regieanweisungen sind dabei zu einem gewissen Grade interpretationsfähig, nie detailliert genug, um eine Situation auszudeuten. Dieser Weg würde nach konkreten, institutionellen Anforderungen an Interaktionsordnungen, Begegnungen, Rollen etc. fragen, die sich aus den beschriebenen Makrodynamiken ergeben. Eine Verbindung dieser Tradition mit dem World-Polity-Ansatz, die konkrete Skripte kollektiven Handelns in lebendiger Praxis rekonstruieren könnte, steht noch aus. 
2.3 Kollektivhandeln, wechselseitige Übersetzung und das Wirken in Praxis ${ }^{73}$

(A) Kollektivhandeln als Prozess wechselseitiger Mobilisierung und wirksame Intervention

Auch wenn Marx im Werke Latours und anderer Vertreter der ANT meist als Prototyp eines materialen Deterministen persifliert wird (siehe etwa Latour 2005: 84), gibt es einen Punkt, an dem die ANT-Bewegung seinem Frühwerk ähnelt: der Fundierung sozialwissenschaftlichen Erklärens im sozio-materialen Prozessieren der Praxis selbst (ebd.: 64). Die bereits bei Marx präsente Grundfigur wechselseitiger Relationierung von Handelnden im Handeln, um handlungsfähig zu sein, belebt sie heute prominent wie keine andere Perspektive im Diskurs. ${ }^{74}$ Handeln ist bei Latour dabei stets kollektives Handeln im Sinne eines Einwirkens als Akteur-Netzwerk. Der Netzwerkbegriff ist hierbei ein metaphorischer. Man kann vom Einwirken in und durch Verbindungen mit anderen Wirkenden sprechen. Für Latour (und andere Autoren der ANT) ist es von zentraler Bedeutung, dass Einwirken auf Welt immer etwas mit einer Relationierung heterogener Elemente (von Körpern, Dingen, Symbolen etc.) zu tun hat, nichts und niemand allein handlungsfähig ist.

73 Siehe für eine ähnliche Auseinandersetzung mit der ANT unter Bezug auf die Materialität der Praxis Schmidt $(2011,2013)$. Bedeutsam schien und scheint es mir, insbesondere die erstaunlich konsistenten Schriften Latours und Laws mit dezidiert sozialtheoretischem Anspruch in das Zentrum zu rücken, wenn man die ANT als spezifische Sozialtheorie diskutiert. Eine Vielzahl an Konzepten mit substantiellem Anspruch von Autoren der ANT-Bewegung zeugen hingegen von einer deutlich größeren Heterogenität in theoretischen Grundfragen.

74 Aus einer Generalisierung der methodologischen und sozialtheoretischen Prinzipien der Laborstudien heraus entstanden, bildet die ANT heute einen vieldiskutierten Ansatz um Kollektivität in einer Vielzahl an Disziplinen und Forschungsfeldern. Aus der interdisziplinären Wissenschafts- und Technikforschung kommend erlebt sie gerade eine beachtenswerte Popularität in der Organisationsforschung, die vor allem durch die Aufnahme der ANT durch etablierte Autoren (bspw. Feldman/Pentland 2003, Czarniewska/Hernes 2005, Gherardi/Nicolini 2005, Orlikowski 2007, Karnoe/Garud 2012) vorangetrieben wird. Die Attraktivität und Parallelen zu mikropolitischen oder Sensemaking-Studien hat bspw. Friedberg (1995) stets gesehen. Vor allem die Grundfigur des ,heterogeneous engineering“ wird dabei in managementorientierten Forschungsbereichen als hilfreiches Konzept diskutiert: „The parallel between the notion of ,heterogeneous engineering' and the concept of ,organizing' makes ANT an understandably attractive theory for scholars of management and organization" (Whittle/Spicer 2008: 612f.). Ob diese Parallele so intendiert ist, lässt sich infrage stellen, stellt der Grundtenor der ANT doch vor allem auf die Einzigartigkeit der Situation und die Ablehnung von Rezeptwissen ab. 
Um auch materiale Elemente mit in dieses zentrale Konzept prozesshaften Verbindens und Verbunden-Seins einbeziehen zu können, verwendet die ANT ein „niedrigschwelliges“ Handlungskonzept, das Konzept von Agency als Erzeugen eines Unterschieds in situ (Latour 2007: 123). Diese begriffliche Wendung ist keineswegs neu und ist dem Informationsbegriff bei Gregory Bateson entlehnt, der unter Information einen „Unterschied, der bei einem späteren Ereignis einen Unterschied ausmacht“ (Bateson 1981: 488, zitiert nach Luhmann 2009: 30) versteht. Nimmt man sich zunächst einen Bestandteil eben dieser Situation, stellt sich vor, dieser wäre nicht mehr vorhanden, und beschreibt dann die Unterschiedlichkeit der so entstandenen, imaginären Situation, hat man auch die Wirkung erfasst, die von eben diesem Bestandteil ausgeht. ${ }^{75}$ Diesen Wirkungsbegriff setzt Latour gleich mit Handeln:

„Soziales Handeln [...] wird auf verschiedene Akteurstypen verlagert oder delegiert, die fähig sind, das Handeln durch andere Aktionsmodi, andere Typen von Materialien zu transportieren“ (ebd.: 122). Delegation heißt dabei, dass , ,jemand/etwas anderes dazu bringt, etwas zu tun“ (ebd.).

Es geht ihm also in seiner Fassung kollektiven Handelns um einen unendlichen Regress des Einwirkens aufeinander. ${ }^{76}$ Handeln ist in diesem Sinne für Vertreter der ANT stets kollektiv, dass man sich gar keine Situation vorzustellen vermag, wo eine Intervention nicht eine gemeinsame ist und somit mit Prozessen wechselseitiger Relationierung zu tun hat. Was immer handelt, ist ein Verbund aus verschiedenen Elementen, ist zugleich ein Akteur und ein Netzwerk verschiedener Akteure (vgl. Callon 1987: 93, Callon/Law 1997: 265). Kollektivhandeln wird zum Handeln in und als spezifische, einander wechselseitig übersetzende Konstellation von Wirkelementen. Die in der Sozialtheorie klassisch vorzufindenden Differenzierungen zwischen Wirken, Verhalten, Handeln sowie sozialem

75 Das spezifisch Andere an Batesons Konzept ist die zeitliche Verzögerung, die bei Batesons die Information ausmacht, also ein Unterschied für ein späteres Ereignis, während Latour das Gedankenexperiment des Beobachters als Lösung vorschlägt.

76 Dies wird eindrucksvoll deutlich, wenn Belliger und Krieger das latoursche Beispiel der Entdeckung des Wirkens von Mikroorganismen durch Pasteur und dem daraus resultierenden Laborbericht kommentieren: „Wenn der Text mehr ist als Phantasie, dann ist der Autor nicht nur der Forscher" (Belliger/Krieger 2006: 32). Experimente werden wiederholt und Wirkungen bleiben gleich. In diesem Sinne schreiben eben auch die Mikroorganismen mit an den großen Entdeckungen der Mikrobiologie. 
und kollektivem Handeln werden hierbei dezidiert negiert, um nicht bereits vorab relevante Elemente der Situation von der Analyse auszuschließen.

(B) Die Konstitution kollektiven Handelns über das Netzwerkbilden in Praxis

Die ANT verfolgt in ihren abstrakten Bestimmungen zur Ontologie eine radikal situationistische Sozialtheorie, die die voraussetzungslose Betrachtung des Wirkens in actu anstelle vorausgesetzter Intentionen oder Institutionen in das Zentrum ihrer Konzeption des Sozialen rückt. ${ }^{77}$ Das heißt aber nicht, dass es keine Stabilisierer oder Ordner gäbe. Bedeutsam scheint den Autoren vielmehr ein performatives Ordnungsverständnis. Die ANT fokussiert auf die wechselseitige Relationierung im praktischen Einwirken aufeinander. Latours Anliegen ist es, den Gesellschaftsbegriff im Begriff der Assoziation aufzulösen, den er symbolisch vom Durkheim-Gegner Gabriel Tarde entlehnt. Latours Vorstellung von performativer Vergesellschaftung referiert in aller Deutlichkeit sein Text über die Mächte der Assoziationen (Latour 2006):

\footnotetext{
„Da [...] die Macht hier und jetzt zusammengesetzt wird, indem viele Akteure in ein gegebenes politisches oder soziales Schema eingebunden werden, und nicht etwas ist, das gelagert und durch eine vorher existierende Gesellschaft an die Mächtigen weitergegeben werden kann, folgt daraus[...], dass die Natur von Gesellschaft verhandelbar, eine praktische und revidierbare Sache (performativ) ist und nicht etwas, das ein für alle Mal von einem Soziologen bestimmt werden kann, der außerhalb zu stehen versucht (ostensiv)“ (ebd.: 195, Herv. RJ).
}

Gesellschaft muss also stets reproduziert werden und besteht aus nichts weiter als den praktisch realisierten Beziehungen unterschiedlicher Komponenten. Derartige Feststellung allein sind sicher keine Alleinstellungsmerkmale des Ansatzes und oftmals fehlt eine ernsthafte Diskussion theoretischer Gegenpositionen. Spezifischer ist die These, dass diese Beziehungen stets verhandelbar sind. Ordnung ist für die ANT immer im Entstehen begriffen, nie einfach vorhanden und (auch für den Soziologen) unmöglich zu fixieren. Das Paradoxon der Macht wird

77 Sie betreibt damit eine nahezu entgegengesetzte Dezentrierung des Subjekts als die FoucaultTradition, eine Konzeption die nicht etwa die Tiefe der Geschichtlichkeit und hinter den Dingen liegende Diskurse betrachtet, sondern vielmehr gerade die Aktualität, das, was im Moment wirkt. 
zu Latours Ausgangspunkt, um in seiner Sozialtheorie einen radikalen „Wechsel vom Prinzip zur Praxis“ (ebd.: 206) zu vollziehen:

„Wenn man einfach nur Macht hat - in potentia -, geschieht nichts und man ist machtlos; wenn man Macht ausübt - in actu -, führen andere die Handlungen aus und nicht man selbst" (ebd.: 196).

Es geht also um eine Fokussierung auf das situative Wirken, eine Wissenschaft des konkret Präsenten. Dies schließt auch eine Abkehr von einer vorab gesetzten, intentionalistischen Deutung von Welt mit ein. Auch die Wirkmächtigkeit von Intentionalität (wie auch Institutionen) muss sich in actu zeigen, zur praktischen Handlungsressource werden (ebd.). Diese Ablehnung von Potentialen, einem Wirken auch in raum-zeitlicher Abwesenheit, das verschiedenen Sozialtheorien innewohnt, macht das Spezifische Verständnis von Welt in der ANT aus. Auch die Aufrechterhaltung kollektiven Handelns würde sodann zu einer Frage der beständig in situ reproduzierten Aktualisierung ,praktischer Ressourcen“.

Die Konstitution kollektiven Handelns wird dabei aus der Versammlung und der wechselseitigen Übersetzung eines Akteur-Netzwerkes und seiner Mobilisierung erklärt. Was sich wechselseitig wirkmächtig macht, muss also zunächst an einem bestimmten Zeitpunkt an einem bestimmten Ort miteinander oder nacheinander versammelt werden, damit es so wirken kann, wie es wirkt. Es geht also um eine Ex-post-Beschreibung von Versammlungs-, Übersetzungs- und Mobilisierungsprozessen. Die Rekonstruktion dieses Prozesses beginnt mit der zentralen methodologischen Forderung nach dem Öffnen der Black Boxes und dem anschließenden „follow the actors““ ${ }^{78}$ Man schaut sich also die Wirkung eines Kollektivs an, öffnet die Black Box, zeichnet nach, welche Akteure in den Beschreibungen der Befragten auftauchen, und versucht ihre jeweiligen Handlungsprogramme und die wechselseitigen Übersetzungsprozesse nachzuzeichnen. Dabei werden eben stets auch Akteure vorausgesetzt, die einander übersetzen.

78 Vgl. instruktiv wie polemisch zum methodischen Vorgehen Latours (2005) Gespräch mit einem Studierenden. 
(C) Die Heterogenität, Prozesshaftigkeit und Banalität des Kollektiven

Die Autoren der ANT und eine Vielzahl anderer Autoren der Ethnomethodologie, des Pragmatismus oder auch verschiedener Praxistheorien beziehen sich auf eine vermeintliche Banalität des Sozialen zurück: dem relationalen Prozessieren der Praxis selbst in ihrem Verlauf in „Echtzeit“ (vgl. Pickering 2001). Erst in Praxis, im Einwirken auf Welt zeigen sich Akteure, Ordnungen und handlungsfähige Kollektive. Die ANT ist hier sicher keine Neuerung, bringt diese Position heute aber äußerst prominent in den sozialtheoretischen Diskurs ein. Sie ist eine der hörbarsten und konsequentesten Stimmen in der Betonung der Fluidität, Instabilität und Heterogenität heutiger Kollektive. Zudem hat sie eine Vielzahl an empirisch angewandten Konzepten prozesshafter Ordnungsbildung entwickelt, etwa das erwähnte „system-building“, die „translation“ und das „enrolement". Unklar ist dabei jedoch, in welcher Relation sie zueinander stehen und inwiefern sie jenseits der referierten Beispiele gelten. Die Autoren verbinden diese Orientierung mit einem weiteren Thema, das die Sozialwissenschaften vermeintlich vergessen haben, dem der Bedeutsamkeit des Materiellen für Ordnungsbildung. Wenngleich ich die Problematisierung einer allgemeinen Materialitätsvergessenheit durch die Autoren nicht Teile, ${ }^{79}$ so werden meines Erachtens, vermittelt über ihre ethnomethodologischen Wurzeln, zwei durchaus bedeutsame Aspekte des Materialen durch die ANT gewürdigt, die bislang in vielen Theorieangeboten, etwa Institutionen- und Handlungstheorien, nicht fokussiert wurden. Hervorgehoben wird zum einen die Bedeutsamkeit von alltäglichen materialen Entitäten, die in anderen Traditionen als Banalitäten häufig aufgrund ihrer Stummheit vergessen werden. Zudem werden den Alltag radikal transformierende Dinge in ihrer Bedeutung im situierten Tun sichtbar.

In Bezug auf die Konstitution haben sie das marxsche Grundthema der Mobilisierung als einem wechselseitigen Prozess der Relationierung wiederbelebt und gezeigt, dass Mobilisierung im Rahmen kollektiven Handelns keineswegs als Investieren von Besitzgütern zu verstehen ist. Vielmehr geht es um die aktive Möglichkeit, praktische Ressourcen einzusetzen. Auch Ressourcen müssen erst in den Prozess eingebracht werden können. Dies hängt nicht nur von den Verfügungsrechten, sondern stets auch von situativen Konstellationen der Dinge,

79 Für eine durchaus plausible Gegenposition in Bezug auf Durkheim siehe Lindemann (2008). 
Kompetenzen und Interessen ab sowie davon, wie diese Konstellation konkret angeordnet und versammelt wird.

(D) Theoretische Herausforderungen eines radikal prozessualen Zugriffs

Sicher ist die ANT eine besonders heterogene Theoriebewegung. Dennoch bezieht sie sich auf einen gemeinsamen ontologischen Kern, den vor allem Latour $(2005,2006)$ mit weitreichendem sozialtheoretischen Anspruch formuliert. Hierbei werden spezifische Antworten auf die klassischen Fragen nach dem Akteursund Handlungsverständnis, sozialer Ordnung und dem Wandel formuliert sowie eine Verortung des Sozialen oder besser Sozio-Materialen angestrebt. Nimmt man die ANT als sozialtheoretische Heuristik ernst, die den eingangs beschriebenen, analytischen Zugriff auf die als neuartig proklamierten Formen des Kollektiven zu informieren vermag, zeigen sich zugleich aber auch einige weitreichende Probleme der Theorieanlage. Sie gründen vor allem in der zentralen methodologischen Forderung einer möglichst unvoreingenommenen Theoriesprache, die Handlungs-, Akteurs-, Wandlungs- und Ordnungsverständnis möglichst symmetrisch für menschliche und nicht-menschliche Elemente $\mathrm{zu}$ fassen versucht. Das Ausflaggen allen Wirkens als kollektiven Handelns im Netzwerk geht zudem mit einer radikalen Negierung der Spezifika des Konzepts und des Phänomens einher.

Hierdurch werden durchaus fruchtbare Thematisierungen menschlicher Vergesellschaftung aus der Sozialtheorie herausgenommen, um Materiales nicht bereits vorab zu einer passiven Bühne zu degradieren, auf der das eigentliche Schauspiel des Zwischenmenschlichen aufgeführt wird. Das Grundproblem an dieser Vorgehensweise ist, dass mit einer derart verallgemeinerten Beschreibungssprache auch eine Vielzahl an Potentialen kritischer Sozialwissenschaft verloren geht. ANT-Studien laufen Gefahr, nur noch das zu betrachten, was im Forschungsfeld längst bekannt ist (siehe Schulz-Schaeffer 2008). Es kommt zu einer detaillierten Reformulierung dessen, was der Fall ist, ohne dahin zu gelangen, was dahintersteckt. Die epistemische Grundposition einer ,ontologische[n] Metaphysik“ (Kneer 2009: 23), die „Auskunft über die kleinsten (letzten) Einheiten [...], aus der sich die ,wirkliche Wirklichkeit" überhaupt zusammensetzt" (ebd.: 21), gibt, hat in ihrem Fokussieren des Wirkens den Kategorien des Feldes keine eigenen entgegenzusetzen. 
In Bezug auf das Konzept des Kollektivhandelns ergeben sich drei weitere, miteinander verbundene Problemlagen:

(i) Eine dezidierte Handlungstheorie und ein Akteursverständnis wird in der ANT zugunsten des Wirkungsbegriffes zurückgewiesen. So wird auch die Abgrenzung Handeln/Verhalten sowohl auf individueller als auch auf kollektiver Ebene zurückgewiesen. Weiterhin wird jedes Wirken als Wirken im Netzwerk bestimmt. Eine theoretische Abgrenzung zwischen individuellem und kollektivem Handeln wird folgerichtig abgelehnt. Damit gehen Potentiale für analytische Bezugspunkte verloren, die andere Ansätze für die Spezifizierung ihrer Heuristiken fruchtbar gemacht haben. Diese werden nicht durch andersartige Bezugspunkte ersetzt. Letztlich geht es ANT-Autoren um die Rekonstruktion eines Prozesses der Mobilisierung verschiedener Elemente (mit verschiedenen Handlungsprogrammen) auf das Erzeugen einer spezifischen Wirkung hin. Das ist der Grundgedanke hinter der methodologischen Forderung, den Akteuren zu folgen. Die ANT legt hiermit im Endeffekt häufig eine zweckrationale Betrachtung kollektiven Handelns an, sie macht dies nur wenig explizit. Es wird von einer spezifischen, gemeinsam erzielten Wirkung ausgegangen. Darüber hinaus werden die am Effekt Beteiligten und ihre wechselseitige Übersetzung rekonstruiert.

(ii) Ein hiermit unmittelbar verknüpftes Problem ist das Verdinglichen von Kollektiven. Die ANT-Bewegung setzt zwar genau an diesem Punkt ihre Kritik an, fordert das radikale „Öffnen“ der Black Boxes kollektiver Akteure, um die zahlreichen, meist banalisierten und unbeobachteten Elemente aufzeigen zu können. Mit ihrem radikalen Fokus auf wechselseitige Relationierung in actu im Zusammenspiel mit der fehlenden Eingrenzung des Handlungsbegriffs bringt sie sich aber ebenso in die Gefahr einer Naturalisierung von Kollektiven ${ }^{80}$ als Black Boxes, da sie sonst in einen unendli-

80 Die prozessuale Konstitution von Kollektivakteuren ist eine der größten Problemlagen prozesstheoretischen Denkens generell. Dies zeigt sich schon bei Marx. Der Marx des 18. Brumaire deutet, jenseits „objektiver“ polit-ökonomischer Positionen, eine relationale Perspektive auf die Überführung einer Klasse an sich in eine Klasse für sich an. Er stellt vor allem auf die Chance (und mitunter auch den Zwang) ab, im Prozess eine bestimmte Position einnehmen zu können bzw. zu müssen. In dieser Lesart marxistischer Tradition sind sowohl kollektive Handlungskapazitäten als auch interessierte Kollektivakteure aus den beständig wechselnden Beziehungen in Verteilungskämpfen zwischen Kollektiven gekennzeichnet(siehe Teil I.1.3.). Die Kollektive 
chen Regress des Black-Box-Öffnens (vgl. Schulz-Schaeffer 2000: 141f.) geraten würde. Was dann in den Studien zumeist geschieht, ist, dass man sich an den Ethno-Kategorien des Feldes orientiert und diejenigen Akteure als Akteure betrachtet, die im Untersuchungsfeld als solche angesehen werden (vgl. zu dieser Kritik Schulz-Schaeffer 2008). In den empirischen Arbeiten wimmelt es sodann von Kollektivakteuren. Aus der eigentlich fruchtbaren Kritik entsteht, durch die dezidierte Ablehnung einer theoretischen Eingrenzung des Akteurs- und Handlungsbegriffs, eine Form der Naturalisierung der Akteursbegriffe des Feldes und somit auch wieder ein Setzen und Stabilisieren konsistenter kollektiver Handlungseinheiten.

(iii) Prinzipiell fokussiert die Theorieanlage auf Kollektivhandeln als zunächst einmaligem Einwirken auf Praxis im Netzwerk. Es wird von einer punktuellen Wirkung ausgegangen und daraufhin den Akteuren gefolgt. Das Netzwerk besteht nur im Einwirken zum Einwirken selbst. Eine dezidierte Konzipierung stabilisierter Kollektive, sowie ihrer Stabilisierung findet sich nicht.

Das letzte Problem verweist direkt auf Problemlagen in der Thematisierung der Konstitution kollektiven Handelns:

(i) Die ANT ist nicht in der Lage, den rückbezüglichen, selbstreferentiellen Charakter sozialer Prozesse aufzunehmen. Die selbst auferlegte Beschränkung auf eine Betrachtung des Sichtbaren, des aktuellen AufeinanderEinwirkens geht mit einer Blindheit in der Verschränkung von Situationen und situationsübergreifenden Dynamiken einher. Strukturen sind in der ANT zugespitzt als Situationen überdauernde Materialität thematisiert, weil sie die Qualifizierung des menschlichen Akteurs als bis zu einem gewissen Grade um Strukturen wissenden Akteur nicht explizieren wollen. Sie tun dies, weil sie eine damit vermeintlich einhergehende Diskriminierung materialer Entitäten befürchten. Die Praxis kollektiven Handelns kann aber nicht verstanden werden, wenn man nicht die darin aktualisierten und sozialisier-

selbst sind jedoch durch ein Interesse und Bewusstsein charakterisiert, die ihrer politökonomischen Position in der kapitalistischen Sozialstruktur erwächst. Marx “ Kollektive bleiben so auch in den praxisbezogenen Studien wie dem Brumaire verdinglichte und stabile Einheiten, die in der Analyse zur Analyse vorausgesetzt werden. 
ten, heterogenen Wissensbestände der handelnden menschlichen Akteure als solche einbezieht und nicht bloß die Wirkungen, die sie zeitigen. Latour (2005, 2006) lehnt wiederholt ostensive, auf Potenzialität und Virtualität rekurrierende Theorien ab und zielt auf eine Vorstellung performativer Vergesellschaftung in actu.

Feldman und Pentland (2003) haben dies am Beispiel organisationaler Routinen und unter Rekurs auf Latours Begriffe und Konzepte zurückgewiesen: Das Soziale hat stets eine performative und ostensive Dimension. Man muss beide aufeinander beziehen und nicht die richtige Fokussierung auf Wirkungen, Performanzen und Vollzug mit einer Ablehnung des Virtuellen, Sozialisierten und Symbolischen verbinden. Jede praktische Ressource, die Latour gern fokussieren würde, wird erst zu einer solchen, wenn sie spezifisch interpretiert, wahrgenommen, sortiert und legitimiert ist. Für all diese ostensiven Aspekte bietet die ANT keinen systematischen Ort in der Sozialontologie an, der ihre Qualitäten jenseits des Performativen zu analysieren erlaubt.

(ii) Unklar ist auch, ob und inwiefern genuin kollektive Handlungsprogramme entstehen können. Sie werden entweder in kleinere Einheiten dekomponiert oder an die Motive eines Systembauers geknüpft, der es versteht, die heterogenen Elemente seinen Zielen entsprechend zu arrangieren. Hierbei ist keineswegs klar, auf welche Vorstellung des Akteurs und des strategischen Handelns sich die ANT bezieht. Zum einen soll es keine Differenzen zwischen Wirken und Handeln geben. Andererseits zeigt sich dennoch eine Art implizite Handlungstheorie, eine mitgeführte Differenz zwischen Wirken und strategisch flexiblem (z.B. Latour 1996) oder ordnendem Handeln (z.B. Law 2006b). Die Verknüpfung hin zum Kollektiven wird so mit einzelnen Konzepten wie denen des Systembauens oder des Enrolement abgefunden, ohne systematisch diskutiert zu werden.

(iii) Weiterhin geht die ANT mitunter von einer Stabilisierung über materiales Überdauern aus, was implizit einer infrastrukturellen Stabilisierung von Kollektiven das Wort redet. ${ }^{81}$ Dies verengt nicht nur die Problematik radi-

81 Vgl. etwa Latours (1996) Beispiel der ordnungsbildenden Funktion des schweren Hotelschlüsselanhängers. Hier nur eine kleine Auswahl für derartige Formulierungen in zentralen Texten der ANT: „Soziale Ordnung würde verschwinden, würden all die alltäglichen materialen Ordner verschwinden, die ständig beteiligt sind, [denn Ordnung ist ein, RJ] durch heterogene Mit- 
kal, es vergisst auch, dass jede Verwendung eines Artefakts auch Wendung desselben beinhaltet (vgl. Orlikowski 2000).

\section{Grundlagen für eine Theorie der Praxis kollektiven Handelns}

Diese Betrachtung klassischer und aktuell bedeutsamer sozialtheoretischer Ansätze liefert wichtige Einsichten. Es wird aber auch deutlich, dass die klassischen Grundfragen kollektiven Handelns nach der Verbindlichkeit eines Kollektivgebildes für das Handeln einzelner Beteiligter (Weber), der Bedeutung geteilter Rahmungen, Zuschreibungen und Klassifikationen von Kollektiven für tatsächliche Kooperation (Durkheim) oder der Mobilisierung geteilter Interessen in einer Gruppe in eine tatsächliche Intervention in Praxis (Marx) weitestgehend getrennt voneinander behandelt werden. Außerdem wird deutlich, dass die bereits bei den Klassikern bestehenden Grundfragen der Konstitution kollektiven Handelns, also Fragen nach der Bedeutung sozialer Ordnungen bzw. sozialer Systeme und gesellschaftsweiten Sichtweisen, der subjektiven Motivation und der konkreten Praxis für kollektives Handeln, weitestgehend getrennt voneinander diskutiert werden. Eine Vermittlung dieser Grundfragen in Praxis über soziale Praktiken ist hierbei ein fruchtbarer Ansatzpunkt, neben fünf weiteren Themenkomplexen, die einer Weiterführung bedürfen:

(i) Insbesondere werden grundlegende Aspekte, die kollektives von individuellem Handeln unterscheiden, diskutiert. Im Gefolge Webers geht es der handlungstheoretischen Tradition um Verbindlichkeit einer Ordnung im und für das individuelle Handeln. Dieses Thema wurde bei Parsons zu einem der affektiven Bindung eines Akteurs an die Werte und Normen eines Kollektivs. Die institutionalistische Tradition argumentiert hingegen für eine Fokussierung auf eine geteilte Weltsicht und objektivierte Formen der Aufnahme und Interpretation von Welt. In dieser Tradition kommt auch der $Z u$ schreibung von Handlungsfähigkeit auf ein Kollektiv zentrale Bedeutung zu. Prozessbezogene Konzepte begreifen die Fähigkeit, sich gegenseitig in ei- 
ner konkreten historischen Situation organisiert zu mobilisieren und in wechselseitiger Relationierung orchestriert wirkmächtig zu werden, als Kern der Handlungsfähigkeit von Kollektiven.

(ii) Unter dem Begriff kollektiven Handelns werden ganz unterschiedliche Phänomene diskutiert. In der ANT steht die situative Kollektivintervention im Zentrum, der Institutionalismus und die Handlungstheorie rückt das Handeln von Kollektivakteuren und die Genese dieser in den Mittelpunkt stellt. Nicht selten ist es forschungspragmatisch sinnvoll, Prozesse des Kollektivhandelns unter Einbezug verschiedener Formen des Kollektivhandelns zu analysieren (siehe bereits Bader 1991).

(iii) Mit einem praxistheoretischen Konstitutionsverständnis geht eine Überführung von in diesen Literaturen thematisierten Dualismen in fünf prozessual vermittelte Spannungsverhältnisse einher, dem zwischen Homogenität und Heterogenität der relevanten Handelnden, Sozialsysteme oder Institutionen; zwischen Reproduktion und Transformation; zwischen Prozessen des Ordnens und dem Verwenden bestehender Ordnungen; zwischen aktiver Regulation und prozessualer Eigendynamik; zwischen Geteiltheit und Durchsetzung einer Kollektivhandeln informierenden Sozialordnung.

Open Access Dieses Kapitel wird unter der Creative Commons Namensnennung 4.0 International Lizenz (http://creativecommons.org/licenses/by/4.0/deed.de) veröffentlicht, welche die Nutzung, Vervielfältigung, Bearbeitung, Verbreitung und Wiedergabe in jeglichem Medium und Format erlaubt, sofern Sie den/die ursprünglichen Autor(en) und die Quelle ordnungsgemäß nennen, einen Link zur Creative Commons Lizenz beifügen und angeben, ob Änderungen vorgenommen wurden.

Die in diesem Kapitel enthaltenen Bilder und sonstiges Drittmaterial unterliegen ebenfalls der genannten Creative Commons Lizenz, sofern sich aus der Abbildungslegende nichts anderes ergibt. Sofern das betreffende Material nicht unter der genannten Creative Commons Lizenz steht und die betreffende Handlung nicht nach gesetzlichen Vorschriften erlaubt ist, ist für die oben aufgeführten Weiterverwendungen des Materials die Einwilligung des jeweiligen Rechteinhabers einzuholen.

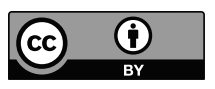

